\title{
Asymptotic Growth of Solutions of Neutral Type Systems
}

\author{
G.M. Sklyar · P. Polak
}

Published online: 7 March 2013

(C) The Author(s) 2013. This article is published with open access at Springerlink.com

\begin{abstract}
We consider a differential system of neutral type with distributed delay. We obtain a precise norm estimation of semigroup generated by the operator corresponding to the system in question. Our result is based on a spectral analysis of the operator and some uniform estimation of norms of the exponentials of matrices. We also discuss the stability properties of corresponding solutions and the existence of the fastest growing solution.
\end{abstract}

Keywords Delay systems $\cdot$ Neutral type systems $\cdot$ Asymptotic behaviour of solutions · Maximal asymptotics

\section{Introduction}

One of the important problems in the theory of functional differential equations is the estimation of the asymptotic behaviour of their solutions. It is also related to stability analysis of those equations. Even in the case when stability is studied, there remains the question about the rate of growth or decay of individual solutions and their dependence on initial states. All those questions pertain directly to the equations with delay. Among the works devoted to this problem, we can single out the works of D.A. Medvedev and V.V. Vlasov [9], W.E. Brumley [3], J.K. Hale, S.M. Verduyn Lunel [4, 5], D.A. O’Connor, T.J. Tarn [11], R. Rabah, G.M. Sklyar [12, 13], R. Rabah et al. [16], S.M. Verduyn Lunel, D.V. Yakubovich [22]. The fundamental approach for estimation of asymptotic growth of solutions is interpretation of

This work was partially supported by Polish National Science Centre grant No. N N514 238438.

G.M. Sklyar $(\bowtie) \cdot$ P. Polak

Institute of Mathematics, University of Szczecin, Wielkopolska 15, 70-451 Szczecin, Poland

e-mail: sklar@univ.szczecin.pl

P. Polak

e-mail: piotr.polak@wmf.univ.szczecin.pl 
the equation as a system in an abstract Banach space and a spectral analysis of the corresponding semigroup. Within this approach traditionally the encountered problem studying is to estimate the norm of the semigroup $\|T(t)\|, t \geq 0$. In the context of asymptotic stability this problem leads to study of so-called exponential stability, because the relation $\|T(t)\| \rightarrow 0, t \rightarrow \infty$, is possible only if $\|T(t)\| \leq M e^{-\lambda t}$ for some positive $M$ and $\lambda$, so the rate of decay of the norm is exponential. This kind of stability is mostly studied for particular classes of equations, for example, for retarded systems by J.K. Hale, S.M. Verduyn Lunel [4], V.B. Kolmanovskii and V.R. Nosov [7]. The strong stability takes an important place in the asymptotic theory of semigroups of last decades, where by strong stability we mean the situation when all the trajectories of the equation $T(t) x, x \in X$, tend to 0 as $t \rightarrow+\infty$ while the norm $\|T(t)\|$ does not decay. Analysis of this kind of stability began in the book of B. Sz.-Nagy, C. Foias, [20] for contractions in Hilbert space and was extended by G.M. Sklyar, V.Ya. Shirman [19] to the case of equations in Banach space. Later it was developed by W. Arendt and C.J.K. Batty [1], Yu.I. Lyubich, V.Q. Phong [8], and then in many other works (see [21] and references therein). Recently some further progress in the analysis of strong stability was achieved, namely we mean some new results concerning the estimation of individual trajectories on nonclosed sets obtained by A. Borichev, Y. Tomilov [2] and some estimations of so-called fast solutions by A. Haraux [6]. Recall that in the case of the strong stability the rate of decay of particular trajectories in general can be arbitrarily slow. Some estimations of this rate are obtained recently by Medvedev, Vlasov, Wu [9, 23]. For delayed systems the effect of the strong stability appears in the case of systems of neutral type. This question was considered in [3], where nonexponential stability for delayed systems was studied. An extensive analysis of this problem was given in [15]. In the recent work [17] a development of the idea of strong stability is proposed. The concept of maximal asymptotics (solution of fastest growth) is introduced there and some conditions under which no such solution exists are obtained. The latter means that there is no $x \in X$ such that $\|T(t) x\| /\|T(t)\| \rightarrow 1, t \rightarrow+\infty$. This concept encouraged us to consider the question of existence of the maximal asymptotics for neutral type equations. Namely it concerns the case when the growth of solutions is lower then the exponential one.

In the present work we consider delay systems of neutral type of the form

$$
\dot{z}(t)=A_{-1} \dot{z}(t-1)+\int_{-1}^{0} A_{2}(\theta) \dot{z}(t+\theta) d \theta+\int_{-1}^{0} A_{3}(\theta) z(t+\theta) d \theta,
$$

where $A_{-1}$ is a $n \times n$ invertible complex matrix, $A_{2}$ and $A_{3}$ are $n \times n$ matrices of functions from $L_{2}(-1,0)$. We develop methods of analysis of stability of neutral type systems proposed in [15]. Now we consider a more general problem-describing the asymptotic behaviour of solutions. Our goal is to estimate solutions' growth speed. Note that recently in [9] an extensive research of a more general class of neutral type equations was given. In particular an upper estimation of solutions' growth of nonhomogenous equation was obtained. In our paper we specify results of [9] for the case of Eq. (1) and we get a more detailed estimation in terms of spectral properties of matrix $A_{-1}$. Our approach allows us to obtain not only the upper estimation of solutions' growth but also the lower estimation of the norm of the corresponding 
semigroup, and the condition under which the upper bound can be reached. Based on those results we consider a problem of null-reachability of a control system connected with (1). Besides, we also give the answer to the question on existence of the fastest growing solution for Eq. (1) (so-called maximal asymptotics, cf. [18]). Following [15] we rewrite Eq. (1) in the operator form

$$
\dot{x}=\mathcal{A} x, \quad x \in M_{2},
$$

where $M_{2}=\mathbb{C}^{n} \times L_{2}\left(-1,0 ; \mathbb{C}^{n}\right)$, the operator $\mathcal{A}$ is then given by

$$
\mathcal{A}\left(\begin{array}{c}
y(t) \\
z_{t}(\cdot)
\end{array}\right)=\left(\begin{array}{c}
\int_{-1}^{0} A_{2}(\theta) \dot{z}_{t}(\theta) d \theta+\int_{-1}^{0} A_{3}(\theta) z_{t}(\theta) d \theta \\
d z_{t}(\theta) / d \theta
\end{array}\right),
$$

and the domain of $\mathcal{A}$ is as follows:

$$
\mathcal{D}(\mathcal{A})=\left\{(y, z(\cdot)): z \in H^{1}\left(-1,0 ; \mathbb{C}^{n}\right), y=z(0)-A_{-1} z(-1)\right\} \subset M_{2} .
$$

Detailed spectral analysis of rewritten system (2)-(4) was presented in [15] and for a more general class of systems in [9]. The crucial role in our further considerations plays the fact that operator $\mathcal{A}$ generates $C_{0}$-semigroup and has finite-dimensional, invariant subspaces which constitute a Riesz basis in $M_{2}$ (see Theorem 16 in [15]).

The work is organized as follows. In Sect. 2 we describe the asymptotic behaviour of the norm of the semigroup generated by the operator $\mathcal{A}$ defined by (3)-(4). We show that the upper and lower bounds of this norm are both of the form $C e^{\omega t}\left(t^{m}+1\right)$, where $\omega, m$ depends only on matrix $A_{-1}$ (Theorem 2.1). We give two examples of Eq. (1), with the same matrix $A_{-1}$ showing that the norm of semigroup can achieve either upper or lower bound. In general, for fixed $A_{-1}$ constant $C$ can not be chosen uniformly with respect to $A_{2}, A_{3}$, unless one includes additional assumptions. However, we prove (Theorem 2.6) that the constant $C$ can be found uniformly with respect to $A_{2}, A_{3}$, if the validity of estimations is required only on some subspace of a finite codimension. The choice of this subspace depends on $A_{2}$ and $A_{3}$.

In Sect. 3 we consider the problem of existence of the fastest growing solution. We study the case when the asymptotic behaviour of the norm of semigroup $e^{\mathcal{A} t}$ is precisely estimated by Theorem 2.1 , namely when matrix $A_{-1}$ has only one Jordan block corresponding to the eigenvalue of maximal modulus. In this case we give the conditions of nonexistence of maximal asymptotics of that semigroup (Theorem 3.1). The result is based on general theorems on the maximal asymptotics $[17,18]$. We also give the explicit form of the maximal asymptotics of Eq. (2) (Theorem 3.2) and discuss it's existence in the general case (Theorem 3.3).

In Sect. 4 we present some applications of obtained results for controlled systems of neutral type. First we consider the feedback control of a special type and give the uniform estimation of semigroup's growth rate (Statement 4.1). Next we estimate the null-reachablility sets of a controlled system under some restrictions on a norm of applied control. Namely we describe the asymptotic behaviour of diameters of those sets (Statement 4.2).

In Appendix we recall some properties of divided differences and prove some statements about special determinants which we use in the proof of auxiliary results (Lemma 2.3). 


\section{Upper and Lower Estimation of the Norm of the Semigroup}

We consider the differential equation given by (2)-(4), which was derived from the neutral type Eq. (1). If $A_{2}$ and $A_{3}$ in (3) equal zero, we denote the operator $\mathcal{A}$ by $\tilde{\mathcal{A}}$ and we add tilde to all the symbols related to this special case (e.g. $\tilde{\lambda}, \tilde{\omega}, \tilde{V}$, etc.). We recall (see Theorems $7,15,16$ [15]) some properties of operators $\mathcal{A}$ and $\tilde{\mathcal{A}}$, which we will need in order to describe the corresponding semigroups. Denote the eigenvalues of the matrix $A_{-1}$ by $\mu_{m}, m=1, \ldots, \ell\left(\left|\mu_{1}\right| \geq\left|\mu_{2}\right| \geq \cdots \geq\left|\mu_{\ell}\right|\right)$, and their multiplicities by $p_{m}\left(\sum p_{m}=n\right)$. The eigenvalues of $\tilde{\mathcal{A}}$ are complex logarithms of $\mu_{m}$ and zero i.e.

$$
\begin{aligned}
\sigma(\tilde{\mathcal{A}})= & \left\{\tilde{\lambda}_{m}^{(k)}=\ln \left|\mu_{m}\right|+i\left(\arg \mu_{m}+2 k \pi\right), \mu_{m} \in \sigma\left(A_{-1}\right), m=1, \ldots, \ell ; k \in \mathbb{Z}\right\} \\
& \cup\{0\} .
\end{aligned}
$$

Almost all eigenvalues of $\mathcal{A}$ lie close to $\tilde{\lambda}_{m}^{(k)}$. More precisely, for $k$ large enough they are contained in the discs $L_{m}^{(k)}$ centered at $\tilde{\lambda}_{m}^{(k)}$ of radiuses $r_{k} \rightarrow 0$ (see Theorem 4 [16]). The sum of multiplicities of eigenvalues of $\mathcal{A}$ lying in each disc centered at $\tilde{\lambda}_{m}^{(k)}$ equals the multiplicity of $\tilde{\lambda}_{m}^{(k)}$ and $\mu_{m}$, that is $p_{m}$. Let us denote $\mathcal{A}$-invariant subspaces $V_{m}^{(k)}=P_{m}^{(k)} M_{2}$, where $P_{m}^{(k)} x=\frac{1}{2 \pi i} \int_{L_{m}^{(k)}} R(\mathcal{A}, \lambda) x d \lambda$ are Riesz projectors, $m=1, \ldots, \ell, k \in \mathbb{Z}$. The sequence of $p_{m}$-dimensional subspaces $V_{m}^{(k)}, m=1, \ldots, \ell,|k| \geq N$, and some $2(N+1) n$-dimensional subspace $W_{N}$ constitute $\mathcal{A}$-invariant Riesz basis of space $M_{2}$. Notice that the subspaces $\tilde{V}_{m}^{(k)}, m=$ $1, \ldots, \ell,|k| \geq N$, and some $2(N+1) n$-dimensional subspace $\tilde{W}_{N}$ constitute $\tilde{\mathcal{A}}$ invariant Riesz basis of the space $M_{2}$, which is quadratically close to the previous one. Theorem 7 states in particular that to each $\tilde{\lambda}_{m}^{(k)} \in \sigma(\tilde{\mathcal{A}}) \backslash\{0\}$ and each Jordan chain of eigen- and rootvectors of the matrix $A_{-1}$ corresponds the Jordan chain of $\tilde{\mathcal{A}}: v_{m, j}^{(k), 0}, v_{m, j}^{(k), 1}, \ldots, v_{m, j}^{(k), p_{m, j}-1}$, i.e. the vectors $v^{s}$ (the indices $k, m, j$ are omitted) satisfy the relation $(\tilde{\mathcal{A}}-\lambda I) v^{s}=v^{s-1}$. They are given by

$$
v_{m, j}^{(k), s}=\left(\begin{array}{c}
0 \\
e^{\lambda_{m}^{(k)} \theta} P_{m, j}^{s}(\theta)
\end{array}\right),
$$

where $P_{m, j}^{s}(\theta)$ is some polynomial independent of $k, s=0, \ldots, p_{m, j}-1 ; m=$ $1, \ldots, \ell ; k \in \mathbb{Z} ; j=1, \ldots, v_{m}$.

To estimate the norm of semigroup generated by the operator $\mathcal{A}$ defined by (3)(4) we have to know how exactly the spectrum of $\mathcal{A}$ looks like. Let us denote $\sup \{\operatorname{Re} \lambda: \lambda \in \sigma(\mathcal{A})\}$ by $\omega$ and $\sup \{\operatorname{Re} \lambda: \lambda \in \sigma(\tilde{\mathcal{A}})\}$ by $\tilde{\omega}$. Notice that $\omega \geq \tilde{\omega}$. The case when $\omega>\tilde{\omega}$ is not interesting because in this case there is only a finite number of eigenvalues of $\mathcal{A}$, whose real parts are close to $\omega$ and thus there is an eigenvalue $\lambda_{0}$ of $\mathcal{A}$ with real part $\omega$. It is easy to see that this eigenvalue will determine the behaviour of semigroup $e^{\mathcal{A} t}$, that is for some positive constants $m, M$ and $t \geq 0$ the inequalities hold

$$
m e^{\omega t}\left(t^{q_{0}-1}+1\right) \leq\left\|e^{\mathcal{A} t}\right\| \leq M e^{\omega t}\left(t^{q_{0}-1}+1\right),
$$

where $1 \leq q_{0} \leq n$ is the maximal size of Jordan block corresponding to $\lambda_{0}$. In the case when $\omega=\tilde{\omega}$ the following theorem describes the behaviour of $\left\|e^{\mathcal{A} t}\right\|$. 
Theorem 2.1 Let us consider a semigroup $e^{\mathcal{A} t}, t \geq 0$, where operator $\mathcal{A}$ is defined by (3)-(4). If $\operatorname{Re} \lambda<\sup \{\operatorname{Re} \tau: \tau \in \sigma(\mathcal{A})\}$ for any $\lambda \in \sigma(\mathcal{A})$ then there exist the constants $M_{p}, M_{q}$, such that the following inequalities hold

(i) $\left\|e^{\mathcal{A} t}\right\| \leq f_{p}(t)=M_{p} e^{\omega t}\left(t^{p-1}+1\right)$,

(ii) $\left\|e^{\mathcal{A} t}\right\| \geq f_{q}(t)=M_{q} e^{\omega t}\left(t^{q-1}+1\right)$,

where $q$ is the maximal size of Jordan block of matrix $A_{-1}$ corresponding to the eigenvalue with maximal modulus and $p$ is the sum of sizes of all Jordan blocks corresponding to the maximal eigenvalue of $A_{-1}$.

Proof (i) In general, there can be more then one eigenvalue of maximal modulus, that is $\left|\mu_{1}\right|=\left|\mu_{2}\right|=\cdots=\left|\mu_{m_{0}}\right|>\left|\mu_{m_{0}+1}\right| \geq \cdots \geq\left|\mu_{\ell}\right|$ for some integer $m_{0} \leq \ell$. Let us denote the sum of sizes of Jordan blocks of matrix $A_{-1}$ corresponding to the eigenvalues $\mu_{1}, \ldots, \mu_{m_{0}}$ by $p_{1}, \ldots, p_{m_{0}}$ respectively, then $p=\max \left\{p_{1}, \ldots, p_{m_{0}}\right\}$. Theorem 4 from [12] implies that there exists $N$ large enough, such that we can split the spectrum of operator $\mathcal{A}$ into three parts. The first part, $\sigma_{N}$, consists of eigenvalues contained in the separated circles $\left\{L_{m}^{(k)}\right\}_{m=1, \ldots, m_{0}}$ with centers in $\tilde{\lambda}_{m}^{(k)}$ and radii $r_{k} \rightarrow$ 0 , such that the number of eigenvalues of $\mathcal{A}$ in a given circle $L_{m}^{(k)}$ equals $p_{m}$ (counting with multiplicities). The second part is called $\sigma_{\varepsilon}$. Therefore we have $\sigma_{N}=\sigma(\mathcal{A}) \cap$ $\bigcup_{m=1, \ldots, m_{0}} \bigcup_{|k|>N} L_{m}^{(k)}$ and $\sigma_{\varepsilon}=\sigma(\mathcal{A}) \backslash \sigma_{N}$. Notice that there exists $\varepsilon>0$ small enough such that every eigenvalue $\lambda \in \sigma_{\varepsilon}$ satisfies $\operatorname{Re} \lambda \leq \omega-\varepsilon$. Denote the images of spectral projectors corresponding to $\sigma_{N}, \sigma_{\varepsilon}$ by $Q_{N}$ and $Q_{\varepsilon}$. Those subspaces are $\mathcal{A}$-invariant and their direct sum equals $M_{2}$. Thus $\left.\mathcal{A}\right|_{Q_{\varepsilon}}$ generates $C_{0}$-semigroup and from Hille-Yosida theorem we have

$$
\left\|e^{\left.\mathcal{A}\right|_{Q_{\varepsilon}} t}\right\| \leq M_{\varepsilon} e^{\omega t}, \quad t \geq 0 .
$$

The operator $\left.\mathcal{A}\right|_{Q_{N}}$ satisfies the assumptions of Theorem 1 in [10] thus it easily follows that

$$
\left\|e^{\left.\mathcal{A}\right|_{Q_{N}} t}\right\| \leq M_{N} e^{\omega t}\left(t^{p-1}+1\right), \quad t \geq 0 .
$$

Subspaces $Q_{N}, Q_{\varepsilon}$ are direct sums of basis subspaces. Thus they also constitute the Riesz basis from subspaces and it is easy to see that there exists a constant $M_{p}$ such that

$$
\left\|e^{\mathcal{A} t}\right\| \leq M_{p} e^{\omega t}\left(t^{p-1}+1\right), \quad t \geq 0 .
$$

(ii) It suffices to show that for any positive $\varepsilon$ and $T$ there exists $x \in M_{2}$ such that for all $t \in[0, T]$ the inequality $\left\|e^{\mathcal{A} t} x\right\| \geq\left(f_{q}(t)-\varepsilon\right)\|x\|$ holds.

Let us denote the maximal size of Jordan block of matrix $A_{-1}$ corresponding to $\mu_{m}$ by $q_{m}$ for $m=1,2, \ldots, m_{0}$, and $\max \left\{q_{1}, \ldots, q_{m_{0}}\right\}$ by $q$. Without loss of generality we assume that $q=q_{1}$. Let us consider $x \in V_{1}^{(k)}$. Later on for simplicity we will omit index 1 and write shortly $V_{k}$ instead of $V_{1}^{(k)}, \lambda_{k}$ instead of $\lambda_{1}^{(k)}, A_{k}$ instead of $A_{1}^{(k)}$ etc. Then $\left\|e^{\mathcal{A} t} x\right\|_{M_{2}}=\left\|e^{A_{k} t} x\right\|_{V_{k}}$, where operator $A_{k}: V_{k} \rightarrow V_{k}$ has eigenvalues close to $\tilde{\lambda}_{k}$. Representing $A_{k}$ as $A_{k}=A_{k}^{0}+\tilde{\lambda}_{k} I$, where $I$ is identity operator, we get for exponents $\left\|e^{A_{k} t} x\right\|_{V_{k}}=\left|e^{\tilde{\lambda}_{k} t}\right|\left\|e^{A_{k}^{0} t} x\right\|_{V_{k}}$. On the other hand $\tilde{\lambda}_{k}$ are the complex 
logarithms of $\mu_{1}$, so $\operatorname{Re} \tilde{\lambda}_{k}=\ln \left|\mu_{1}\right|=\tilde{\omega}=\omega$ and our inequality can be rewritten in the form

$$
\left\|e^{\mathcal{A} t} x\right\|_{M_{2}}=e^{\omega t}\left\|e^{A_{k}^{0} t} x\right\|_{V_{k}} .
$$

Because the families of subspaces $\left\{V_{m}^{(k)}\right\}_{k \in \mathbb{Z} ; m=1, \ldots, \ell}$ and $\left\{\tilde{V}_{m}^{(k)}\right\}_{k \in \mathbb{Z} ; m=1, \ldots, \ell}$ constitute quadratically close Riesz basis, there exists a bounded operator $T_{N}$, with bounded inverse, which is close to identity and transforms almost all subspaces $V_{m}^{(k)}$ to $\tilde{V}_{m}^{(k)}$. Such operator $T_{N}$ can be defined on every basis subspace $V_{m}^{(k)}$ by the formula

$$
\left.T_{N}\right|_{V_{m}^{(k)}} x=\tilde{P}_{m}^{(k)} x, \quad \text { for }|k|>N, m=1,2, \ldots, \ell,
$$

where $\tilde{P}_{m}^{(k)}: V_{m}^{(k)} \rightarrow \tilde{V}_{m}^{(k)}$ is a Riesz projector given by

$$
\tilde{P}_{m}^{(k)} x=\frac{1}{2 \pi i} \int_{L_{m}^{(k)}} R(\tilde{\mathcal{A}}, \lambda) x d \lambda
$$

and

$$
\left.T_{N}\right|_{V_{m}^{(k)}} x=x, \quad \text { for }|k| \leq N, m=1,2, \ldots, \ell .
$$

It is easy to see that the operator $T_{N}$ is bounded on $M_{2}$ and close to identity. Therefore $T_{N}$ is invertible, its inverse $T_{N}^{-1}$ is bounded and transforms all but finitely many subspaces $V_{m}^{(k)}$ onto $\tilde{V}_{m}^{(k)}$. Using $T_{N}$ we can define the operator $\mathcal{B}_{k}: \tilde{V}_{k} \rightarrow \tilde{V}_{k}$ close to $A_{k}^{0}: V_{k} \rightarrow V_{k}$ for each $|k|>N$ by the formula $\mathcal{B}_{k}=T_{N} A_{k}^{0} T_{N}^{-1}$. Let $\tilde{x}=T_{N} x$. Then we get

$$
\left\|e^{\mathcal{B}_{k} t} \tilde{x}\right\|=\left\|T_{N} e^{A_{k}^{0} t} x\right\| \leq\left\|T_{N}\right\|\left\|e^{A_{k}^{0} t} x\right\| .
$$

From here and (9) we obtain

$$
\left\|e^{\mathcal{A} t} x\right\|_{M_{2}} \geq\left\|T_{N}\right\|^{-1} e^{\omega t}\left\|e^{\mathcal{B}_{k} t} \tilde{x}\right\|_{\tilde{V}_{k}} .
$$

The eigen- and rootvectors of operator $\tilde{\mathcal{A}}$ are given by (5), we can see that the number and lengths of all Jordan chains of operators $\tilde{A}_{k}=\left.\tilde{\mathcal{A}}\right|_{\tilde{V}_{k}}$ are independent of $k$. Thus all operators $\tilde{A}_{k}^{0}:=\tilde{A}_{k}-\tilde{\lambda}_{k} I$ have the same matrix, say $A_{0}$ in the basis $\left\{v^{(k), s}\right\}_{s=1}^{p_{1}}$. We will now compare $\tilde{A}_{k}^{0}$ to each $\mathcal{B}_{k}$. Using the variation of a constant to a system

$$
\left\{\begin{array}{l}
\dot{y}=\tilde{A}_{k}^{0} y+\left(\mathcal{B}_{k}-\tilde{A}_{k}^{0}\right) y, \\
y(0)=\tilde{x}
\end{array}\right.
$$

we obtain

$$
e^{\mathcal{B}_{k} t} \tilde{x}=e^{\tilde{A}_{k}^{0} t} \tilde{x}+\int_{0}^{t} e^{\tilde{A}_{k}^{0}(t-\tau)}\left(\mathcal{B}_{k}-\tilde{A}_{k}^{0}\right) e^{\mathcal{B}_{k} \tau} \tilde{x} d \tau .
$$

Hence

$$
\left\|e^{\mathcal{B}_{k} t} \tilde{x}-e^{\tilde{A}_{k}^{0} t} \tilde{x}\right\| \leq\left\|\mathcal{B}_{k}-\tilde{A}_{k}^{0}\right\| \int_{0}^{t}\left\|e^{\tilde{A}_{k}^{0}(t-\tau)}\right\|\left\|e^{\mathcal{B}_{k} \tau} \tilde{x}\right\| d \tau,
$$


for any $|k|>N$. Let us define a family of operators $S_{k}: \tilde{V}_{k} \rightarrow \mathbb{C}^{p_{1}}$ by the formula $S_{k} \tilde{x}_{k}=\alpha^{(k)}$, where $\alpha^{(k)}=\left[\alpha_{1}^{(k)}, \ldots, \alpha_{p_{1}}^{(k)}\right]$ is the vector of coefficients of $\tilde{x}_{k}$ in the basis $\left\{v_{i}^{(k)}\right\}_{i=1}^{p_{1}}$. It is easy to see that each $S_{k}$ is invertible. Using the fact that Jordan chains of operator $\tilde{\mathcal{A}}$ constitute the Riesz basis in the closure of their linear span it is also easy to show that the norms of $S_{k}, S_{k}^{-1}$ are uniformly bounded. With this notation (14) reads

$$
\left\|e^{\mathcal{B}_{k} t} \tilde{x}-e^{\tilde{A}_{k}^{0} t} \tilde{x}\right\| \leq\left\|\mathcal{B}_{k}-\tilde{A}_{k}^{0}\right\| \int_{0}^{t}\left\|S_{k} e^{A_{0}(t-\tau)} S_{k}^{-1}\right\|\left\|S_{k} e^{B_{k} \tau} S_{k}^{-1} \tilde{x}\right\| d \tau,
$$

where $B_{k}=S_{k}^{-1} \mathcal{B}_{k} S_{k}$. To better elaborate the estimation (15) we need the following lemmas.

Lemma 2.2 For every $\delta>0$ there exists $k_{0}$ large enough, that for every $|k|>k_{0}$ we have $\left\|\mathcal{B}_{k}-\tilde{A}_{k}^{0}\right\|<\delta$.

Proof Let us take any $\tilde{x} \in \tilde{V}_{k},|k|>N$ and estimate $\left\|\mathcal{B}_{k} \tilde{x}-\tilde{A}_{k}^{0} \tilde{x}\right\|$. From the definition of $\mathcal{B}_{k}$ we have $\mathcal{B}_{k} \tilde{x}=\left.\left.T_{N}\right|_{V_{k}} A_{k}^{0} T_{N}^{-1}\right|_{\tilde{V}_{k}} \tilde{x}$, where $T_{N}$ is defined by (10)(11). We get $\left\|\mathcal{B}_{k} \tilde{x}-\tilde{A}_{k}^{0} \tilde{x}\right\|=\left\|\tilde{P}_{k} A_{k}^{0} x-\tilde{A}_{k}^{0} \tilde{P}_{k} x\right\|$, where $x=\left.T_{N}^{-1}\right|_{\tilde{V}_{k}} \tilde{x}$. One can now extend operators $A_{k}^{0}$ and $\tilde{A}_{k}^{0}$ to the whole space $M_{2}$ by formulas $\bar{A}_{k}^{0} x=$ $\frac{1}{2 \pi i} \int_{L_{k}}\left(\lambda-\lambda_{k}\right) R(\mathcal{A}, \lambda) x d \lambda$ and $\hat{A}_{k}^{0} x=\frac{1}{2 \pi i} \int_{L_{k}}\left(\lambda-\lambda_{k}\right) R(\tilde{\mathcal{A}}, \lambda) x d \lambda$. It is easy to see that $\left.\bar{A}_{k}^{0}\right|_{V_{k}}=A_{k}^{0}$ and $\left.\hat{A}_{k}^{0}\right|_{\tilde{V}_{k}}=\tilde{A}_{k}^{0}$. Using this and the fact that $\tilde{A}_{k}^{0} \tilde{P}_{k}=\hat{A}_{k}^{0} \tilde{P}_{k}=\tilde{P}_{k} \hat{A}_{k}^{0}$ we have $\left\|\mathcal{B}_{k} \tilde{x}-\tilde{A}_{k}^{0} \tilde{x}\right\|=\left\|\tilde{P}_{k}\left(\bar{A}_{k}^{0}-\hat{A}_{k}^{0}\right) x\right\|$. Notice that the Riesz projectors $\tilde{P}_{k}$ are uniformly bounded by some constant $C$ and $\left\|\bar{A}_{k}^{0}-\hat{A}_{k}^{0}\right\|$ can be estimated by $\left\|\bar{A}_{k}^{0}-\hat{A}_{k}^{0}\right\| \leq r \sup _{\lambda \in L_{k}}\|R(\mathcal{A}, \lambda)-R(\tilde{\mathcal{A}}, \lambda)\|$. It is shown in [15] that such a difference of resolvents satisfies

$$
\sup _{\lambda \in L_{k}}\|R(\mathcal{A}, \lambda)-R(\tilde{\mathcal{A}}, \lambda)\| \leq \gamma_{k}
$$

where $\gamma_{k} \rightarrow 0$ and $r:=\sup \left\{r_{k}\right\}$ is a fixed radius of all $L_{m}^{(k)}$, thus we obtain $\left\|\mathcal{B}_{k} \tilde{x}-\tilde{A}_{k}^{0} \tilde{x}\right\| \leq \operatorname{Cr} \gamma_{k}\|\tilde{x}\| \leq \delta\|\tilde{x}\|$. This completes the proof of Lemma 2.2.

Lemma 2.3 Let $A_{0} \in M_{n}(\mathbb{C})$ be a matrix of Jordan form of eigenvalue 0 , and let $\mathcal{F}$ be any family of matrices from a neighbourhood of $A_{0}$ t.i. $\mathcal{F} \subset U_{\delta}\left(A_{0}\right)=\{A \in$ $\left.M_{n}(\mathbb{C}):\left\|A-A_{0}\right\|<\delta\right\}$. Then there exists such a constant $M_{\delta}$, that for all $A \in \mathcal{F}$

$$
\left\|e^{A t}\right\| \leq M_{\delta} e^{\omega t}\left(t^{n-1}+1\right)
$$

where $\omega=\sup _{A \in \mathcal{F}} \max _{\lambda \in \sigma(A)} \operatorname{Re} \lambda$.

Proof Without loss of generality we will assume that every $A \in \mathcal{F}$ has $n$ different eigenvalues or we can replace such matrix by the sequence of matrices with different eigenvalues tending to matrix $A$. For any matrix $A \in \mathcal{F}$ we can write $e^{A t}$ as a finite 
sum of powers of $A$ with polynomial coefficients $\alpha_{k}^{A}(t)$ and estimate its norm by triangle inequality,

$$
\left\|e^{A t}\right\| \leq \sum_{k=0}^{n-1}\left|\alpha_{k}^{A}(t)\right| \cdot\left\|A^{k}\right\| .
$$

There exists a constant $m(n, \delta)$ such for every Jordan form matrix $A_{0}$ of eigenvalue 0 and for every $A \in \mathcal{F}$ satisfies $\left\|A^{k}\right\| \leq m(n, \delta)$ for each $k=0, \ldots, n-1$. Using this we get the estimation

$$
\left\|e^{A t}\right\| \leq m \sum_{k=0}^{n-1}\left|\alpha_{k}^{A}(t)\right| .
$$

Now we apply Statement 1 and Statement 2 of Appendix to determinant $\alpha_{k}^{A}(t)$ and expand it along $(k+1)$-th row,

$$
\left|\alpha_{k}^{A}(t)\right|=\left|\sum_{i=1}^{n}(-1)^{k+1+i} t^{i-1} f\left[\lambda_{i} t, \ldots, \lambda_{1} t\right] P_{i}^{(k)}(\lambda)\right|,
$$

where $P_{i}^{(k)}(\lambda), i, k \in\{1, \ldots, n\}$ are polynomials (independent of $A$ ) of variables $\lambda_{1}, \ldots, \lambda_{n}$ and $f\left[\lambda_{i} t, \ldots, \lambda_{1} t\right]$ is a divided difference of $i$-th order of function $f(z)=e^{z}$. We estimate $\left|\alpha_{k}^{A}(t)\right|$ using triangle inequality,

$$
\left|\alpha_{k}^{A}(t)\right| \leq \sum_{i=1}^{n} t^{i-1}\left|f\left[\lambda_{i} t, \ldots, \lambda_{1} t\right]\right| \cdot\left|P_{i}^{(k)}(\lambda)\right| .
$$

At the right-hand side we increase $t^{i-1}$ to $\left(t^{n-1}+1\right)$ and use Property 3 of divided differences (see Appendix), obtaining

$$
\left|\alpha_{k}^{A}(t)\right| \leq e^{\omega t}\left(t^{n-1}+1\right) \sum_{i=1}^{n}\left|f\left[\left(\lambda_{i}-\omega\right) t, \ldots,\left(\lambda_{1}-\omega\right) t\right]\right| \cdot\left|P_{i}^{(k)}(\lambda)\right| .
$$

Terms $\left(\lambda_{i}-\omega\right)$ are in $\mathbb{C}^{-}$for $i=1, \ldots, n$, thus we can use Property 2 of Appendix which states that there exists such a constant $M$, that $\left|f\left[\left(\lambda_{i}-\omega\right) t, \ldots,\left(\lambda_{1}-\omega\right) t\right]\right|<$ $M$ for $i=1, \ldots, n$. Let us denote the closure of the set $\bigcup_{A \in \mathcal{F}} \sigma(A)$ by $\sigma_{F}$. Set $\sigma_{F}$ is compact, so the family of polynomials $P_{i}^{(k)}(\lambda)$ is bounded on $\prod_{j=1}^{n} \sigma_{F} \subset \mathbb{C}^{n}$. Let $P=\max _{k, i} \max _{\lambda}\left|P_{i}^{(k)}(\lambda)\right|$. Then we have the estimation

$$
\left|\alpha_{k}^{A}(t)\right| \leq e^{\omega t}\left(t^{n-1}+1\right) M P \sum_{i=1}^{n} 1=e^{\omega t}\left(t^{n-1}+1\right) M P n .
$$

Applying it to (17) we get

$$
\left\|e^{A t}\right\| \leq m \sum_{k=0}^{n-1}\left|\alpha_{k}^{A}(t)\right| \leq m \sum_{k=0}^{n-1} e^{\omega t}\left(t^{n-1}+1\right) M P n=e^{\omega t}\left(t^{n-1}+1\right) m M P n^{2} .
$$


Denoting $M_{\delta}=m M P n^{2}$ we have the estimation

$$
\left\|e^{A t}\right\| \leq M_{\delta} e^{\omega t}\left(t^{n-1}+1\right)
$$

which concludes the proof of Lemma 2.3.

Proof of Theorem 2.1 (cont.) Let us now fix time $T>0$ and $\varepsilon>0$. It is obvious that $\left\|e^{A_{0}(t-\tau)}\right\|$ and $\left\|e^{\mathcal{B}_{k} \tau}\right\|$ are bounded uniformly for $\tau \in[0, t], t \in[0, T]$. From Lemma 2.2 the family of matrices $B_{k}$ is close to matrix $A_{0}$, hence it satisfies assumptions of Lemma 2.3. Norms $\left\|e^{B_{k} \tau}\right\|$ are also bounded uniformly with respect to $k$, thus we can rewrite inequality (15) in the form

$$
\left\|e^{\mathcal{B}_{k} t} \tilde{x}-e^{\tilde{A}_{k}^{0} t} \tilde{x}\right\| \leq M_{T}\left\|\mathcal{B}_{k}-\tilde{A}_{k}^{0}\right\|\|\tilde{x}\|, \quad t \in[0, T],
$$

where constant $M_{T}$ is independent of $k$. Using the above and (13) we obtain

$$
\left\|e^{\mathcal{A} t} x\right\|_{M_{2}} \geq\left\|T_{N}\right\|^{-1} e^{\omega t}\left(\left\|e^{\tilde{A}_{k}^{0} t} \tilde{x}\right\|-M_{T}\left\|\mathcal{B}_{k}-\tilde{A}_{k}^{0}\right\|\|\tilde{x}\|\right) .
$$

Now, let us assume that $x \in V_{k}$ is of the form $x:=T_{N}^{-1} \tilde{x}_{k}^{q}$, where $\tilde{x}_{k}^{q} \in \tilde{V}_{k}$ is the rootvector of $\tilde{A}_{k}$ of the maximal order $q$. That means $\left\|e^{\tilde{A}_{k}^{0} t} \tilde{x}_{k}^{q}\right\|^{2}=\| e^{0 \cdot t}\left(\tilde{x}_{k}^{q}+\right.$ $\left.t \tilde{x}_{k}^{q-1}+\cdots+\frac{t^{q-1}}{(q-1) !} \tilde{x}_{k}^{1}\right) \|^{2}$. Jordan chains of operator $\tilde{\mathcal{A}}$ constitute a Riesz basis in $M_{2}$ (see Remark 9 in [15]) so there exists a constant $c>0$ such that $\left\|\tilde{x}_{k}^{q}+t \tilde{x}_{k}^{q-1}+\cdots+\frac{t^{q-1}}{(q-1) !} \tilde{x}_{k}^{1}\right\|^{2} \geq c^{2}\left(\left\|\tilde{x}_{k}^{q}\right\|^{2}+\left\|t \tilde{x}_{k}^{q-1}\right\|^{2}+\cdots+\left\|\frac{t^{q-1}}{(q-1) !} \tilde{x}_{k}^{1}\right\|^{2}\right)$. Thus we have $\left\|e^{\tilde{A}_{k}^{0} t} \tilde{x}_{k}^{q}\right\|^{2} \geq c^{2}\left(\left\|\tilde{x}_{k}^{q}\right\|^{2}+\left\|\frac{t^{q-1}}{(q-1) !} \tilde{x}_{k}^{1}\right\|^{2}\right)$. Notice also that $\left\|\tilde{x}_{k}^{1}\right\|, \ldots,\left\|\tilde{x}_{k}^{q}\right\|$ are independent of $k$ (see Theorem 7 and Remark 9 in [15]), which gives us $\left\|e^{\tilde{A}_{k}^{0} t} \tilde{x}_{k}^{q}\right\|^{2} \geq$ $c^{2}\left(\left\|\tilde{x}_{k}^{q}\right\|^{2}+\left|\frac{t^{q-1} c_{1}}{(q-1) !}\right|^{2}\left\|\tilde{x}_{k}^{q}\right\|^{2}\right)$ for some constant $c_{1}$, again independent of $k$. Reassuming, we have

$$
\left\|e^{\tilde{A}_{k}^{0} t} \tilde{x}_{k}^{q}\right\| \geq c\left(1+t^{q-1}\right)\left\|\tilde{x}_{k}^{q}\right\|
$$

for some new positive constant $c$ independent of $k$. Inserting $x=x_{k}^{q}=T_{N}^{-1} \tilde{x}_{k}^{q}$ to (19) and using the above we conclude that

$$
\left\|e^{\mathcal{A} t} x_{k}^{q}\right\|_{M_{2}} \geq\left\|T_{N}\right\|^{-1} e^{\omega t}\left(c\left(1+t^{q-1}\right)-M_{T}\left\|\mathcal{B}_{k}-A_{0}\right\|\right)\left\|\tilde{x}_{k}^{q}\right\| .
$$

Denoting constant $c\left\|T_{N}\right\|^{-1}$ by $M_{q}$ and choosing $\delta=\frac{\varepsilon\left\|T_{N}\right\|}{M_{T}} \min \left\{1, e^{-\omega T}\right\}$ we obtain by Lemma 2.2

$$
\left\|e^{\mathcal{A} t} x_{k}^{q}\right\|_{M_{2}} \geq\left(f_{q}(t)-\varepsilon\right)\left\|x_{k}^{q}\right\|, \quad t \in[0, T],|k|>k_{0},
$$

and the proof of Theorem 2.1 is complete.

Remark 1 Recently an upper bound of solutions' growth rate of a general nonhomogeneous equation was given in [9], see Theorem 7.4.1. In the case of Eq. (2) it takes the form $\|x(t)\| \leq M e^{\omega t} t^{N_{0}}$, where $N_{0}$ is a generally unknown constant, which depends on the location of the spectrum of operator $\mathcal{A}$. In this context we would 
like to note that our result (Theorem 2.1(i)) concretizes those estimations. Besides, our approach allows us to obtain also a lower bound of the norm of a corresponding semigroup of matrix $A_{-1}$.

Remark 2 In Theorem 2.1 terms $\left(t^{p-1}+1\right)$ and $\left(t^{q-1}+1\right)$ can be replaced by $t^{p-1}$ and $t^{q-1}$ but the estimation will be valid for $t>1$.

Corollary 2.4 If we omit the assumption that $\operatorname{Re} \lambda<\omega, \lambda \in \sigma(\mathcal{A})$, then using the same methods as in the proof of Theorem 2.1 we can prove that there exist positive constants $m, M$ such that

(i) $\left\|e^{\mathcal{A} t}\right\| \leq M e^{\omega t} t^{\max \left\{p, q_{0}\right\}-1}, t>1$,

(ii) $\left\|e^{\mathcal{A} t}\right\| \geq m e^{\omega t} t^{\max \left\{q, q_{0}\right\}-1}, t>1$,

where $q_{0}$ is the maximal size of Jordan block corresponding to eigenvalues $\lambda \in \sigma(\mathcal{A})$ : $\operatorname{Re} \lambda=\omega$.

Without assumption $\operatorname{Re} \lambda<\omega, \lambda \in \sigma(\mathcal{A})$ we can not estimate the multiplicity of eigenvalues of $\mathcal{A}$ with real parts equal $\omega$ in terms of matrix $A_{-1}$. If we take $\max \left\{p, q_{0}\right\}$ instead of $p$ in the proof of Theorem 2.1 we get assertion (i). Assertion (ii) follows from the fact that in the proof of Theorem 2.1 we did not use assumption $\operatorname{Re} \lambda<\omega$ and from validity of the estimation $\left\|e^{\mathcal{A} t}\right\| \geq m e^{\omega t} t^{q_{0}-1}, t>1$.

Corollary 2.5 If matrix $A_{-1}$ has only one Jordan block corresponding to the eigenvalue of maximal modulus, that is $p=q$, then the norm of the semigroup $e^{\mathcal{A} t}$ behaves exactly like the function $f_{q}$ in the sense $f_{q}(t) \leq\left\|e^{\mathcal{A} t}\right\| \leq C f_{q}(t)$ for some constant $C$.

In general, $p \neq q$ and the estimation given in Theorem 2.1 is not precise, the upper and lower estimations differ by degree of the polynomial. The following examples show that for a fixed $A_{-1}$ the both estimations, upper and lower, can be reached for some $A_{2}, A_{3}$. It means that in general Theorem 2.1 cannot be improved without further assumptions on $A_{2}, A_{3}$.

Example 2.1 We consider Eq. (1) with $A_{-1}=I, A_{2}=f(\theta) I, A_{3}=g(\theta) I$, where $f, g \in L_{2}(-1,0)$ and $I=\left(\begin{array}{ll}1 & 0 \\ 0 & 1\end{array}\right)$. We show that there exist functions $f, g$ such that the lower estimation from Theorem 2.1 is reached. Equation (1) has the form

$$
\dot{z}(t)=I\left(\dot{z}(t-1)+\int_{-1}^{0} f(\theta) \dot{z}(t+\theta) d \theta+\int_{-1}^{0} g(\theta) z(t+\theta) d \theta\right) .
$$

Matrix $A_{-1}$ has one eigenvalue $\mu_{1}=1$ of multiplicity $p_{1}=2$, thus operator $\tilde{\mathcal{A}}$ has a sequence of eigenvalues of the form $\left\{\tilde{\lambda}_{1}^{k}=2 k \pi i\right\}_{k \in \mathbb{Z}}$. If $z=\left(z^{(1)}, z^{(2)}\right)^{T}$, then the Eq. (20) can be splitted into two independent equations,

$$
\left\{\begin{array}{l}
\dot{z}^{(1)}(t)=\dot{z}^{(1)}(t-1)+\int_{-1}^{0} f(\theta) \dot{z}^{(1)}(t+\theta) d \theta+\int_{-1}^{0} g(\theta) z^{(1)}(t+\theta) d \theta \\
\dot{z}^{(2)}(t)=\dot{z}^{(2)}(t-1)+\int_{-1}^{0} f(\theta) \dot{z}^{(2)}(t+\theta) d \theta+\int_{-1}^{0} g(\theta) z^{(2)}(t+\theta) d \theta
\end{array}\right.
$$


where each equation is a scalar version of Eq. (1). Using Theorem 8 from [16] to both scalar equations we get that for any sequence $\tau^{(k)}$ quadratically close to sequence $\tilde{\lambda}_{1}^{(k)}$ there are such functions $f, g$ that $\lambda_{1}^{(k)}=\tau^{(k)}$. Hence in the case of Eqs. (21) there exist functions $f, g$ such that the spectrum $\lambda_{1}^{(k)}$ of corresponding operator $\mathcal{A}$ is in the left open halfplane, thus assumptions of Theorem 2.1 are satisfied. In this case $p=2$ and $q=1$ so the estimation

$$
M_{q} \leq\left\|e^{\mathcal{A} t}\right\| \leq M_{p}(t+1)
$$

from Theorem 2.1 is not precise. On the other hand it is clear that there exists a Riesz basis consisted of eigenvectors of operator $\mathcal{A}$. Thus it is easy to see that $\left\|e^{\mathcal{A} t}\right\| \leq M_{1}$. Hence we proved that there exist functions $f, g$ such that $M_{q} \leq\left\|e^{\mathcal{A} t}\right\| \leq M_{1}$, which means that the lower estimation from Theorem 2.1 can be reached.

Example 2.2 We consider (1) with the same matrix $A_{-1}=I$ and $A_{2}=f(\theta)\left(\begin{array}{ll}1 & 1 \\ 0 & 1\end{array}\right)$, $A_{3}=g(\theta)\left(\begin{array}{ll}1 & 1 \\ 0 & 1\end{array}\right), f, g \in L_{2}(-1,0)$. Now Eq. (1) has the form

$$
\dot{z}(t)=\dot{z}(t-1) I+\left(\begin{array}{ll}
1 & 1 \\
0 & 1
\end{array}\right)\left(\int_{-1}^{0} f(\theta) \dot{z}_{t}(\theta) d \theta+\int_{-1}^{0} g(\theta) z_{t}(\theta) d \theta\right) .
$$

The corresponding characteristic function $\Delta$ is of the form

$$
\begin{aligned}
\Delta(\lambda)=\operatorname{det}\left[I\left(\lambda e^{-\lambda}+\lambda \int_{-1}^{0} f(s) e^{\lambda s} \mathrm{~d} s+\int_{-1}^{0} g(s) e^{\lambda s} \mathrm{~d} s-\lambda\right)\right. \\
\left.+\left(\begin{array}{ll}
0 & 1 \\
0 & 0
\end{array}\right)\left(\lambda \int_{-1}^{0} f(s) e^{\lambda s} \mathrm{~d} s+\int_{-1}^{0} g(s) e^{\lambda s} \mathrm{~d} s-\lambda\right)\right],
\end{aligned}
$$

and it equals zero only if

$$
\lambda=\lambda e^{-\lambda}+\lambda \int_{-1}^{0} f(s) e^{\lambda s} d s+\int_{-1}^{0} g(s) e^{\lambda s} d s .
$$

Once again there exist functions $f, g$ such that all eigenvalues of corresponding operator $\mathcal{A}$ are in the open left halfplane, quadratically close to $\left\{\tilde{\lambda}_{1}^{k}=2 k \pi i\right\}_{k \in \mathbb{Z}}$ and each one has a two-dimensional Jordan block. Thus we can easily show that $\left\|e^{\mathcal{A} t}\right\|$ behaves like a function $M(t+1)$ i.e. there exist positive constants $C_{1}, C_{2}$ such that $C_{1}(t+1) \leq\left\|e^{\mathcal{A} t}\right\| \leq C_{2}(t+1)$.

The examples above show that for fixed matrix $A_{-1}$ and proper choice of $A_{2}, A_{3}$ the top or bottom estimation of the norm of semigroup $e^{\mathcal{A} t}$ can be reached. One can ask if the constants $M_{p}, M_{q}$ can be chosen independently on $\mathcal{A}$. The answer is negative in general. Next theorem states that constants $M_{p}, M_{q}$ can be chosen uniformly, but the estimations are valid for initial states from subspace of $M_{2}$ of finite codimension $2(N+1) n$ (see [15] Theorem 15), which depends on $\mathcal{A}$, namely we have 
Theorem 2.6 Let us consider a semigroup $e^{\mathcal{A} t}, t \geq 0$, where operator $\mathcal{A}$ is defined by (3)-(4). If we assume that $\operatorname{Re} \lambda<\sup \{\operatorname{Re} \lambda: \lambda \in \sigma(\mathcal{A})\}$ for any $\lambda \in \sigma(\mathcal{A})$ then there exist the constants $M_{q}, M_{p}$, which are independent of $A_{2}, A_{3}$ such that the following inequalities hold

1. $\left\|e^{\mathcal{A} t} x\right\| \leq f_{p}(t)\|x\|=M_{p} e^{\omega t}\left(t^{p-1}+1\right)\|x\|, x \in M_{2}^{(N)}$,

2. $\left\|e^{\mathcal{A} t}\right\|_{M_{2}^{(N)}} \geq f_{q}(t)=M_{q} e^{\omega t}\left(t^{q-1}+1\right)$,

where $M_{2}^{(N)}$ is some $\mathcal{A}$-invariant subspace of $M_{2}$ of finite codimension $2(N+1) n$, $q$ is the maximal size of Jordan block of matrix $A_{-1}$ corresponding to eigenvalue $\mu_{1}$ and $p$ is the sum of sizes of all Jordan blocks corresponding to $\mu_{1}$.

Proof For any $\mathcal{A}$, subspaces $\left\{V_{m}^{(k)}\right\}_{m=1, \ldots, \ell ;|k|>N}$ constitute the Riesz basis in their linear span, say $M_{2}^{(N)}$. Thus for any $x \in M_{2}^{(N)}$ we have $x=\sum_{m=1}^{\ell} \sum_{|k|>N} x_{m}^{(k)}$, $x_{m}^{(k)} \in V_{m}^{(k)}$, and

$$
c_{A} \sum_{\substack{m=1, \ldots, \ell \\|k|>N}}\left\|x_{m}^{(k)}\right\|^{2} \leq\|x\|^{2} \leq C_{A} \sum_{\substack{m=1, \ldots, \ell \\|k|>N}}\left\|x_{m}^{(k)}\right\|^{2}, \quad x_{m}^{(k)} \in V_{m}^{(k)} .
$$

We will show that for any $\mathcal{A}$ there exists $N$ such that the constants $C_{A}$ and $1 / c_{A}$ are uniformly bounded with respect to $A_{2}, A_{3}$. Indeed the basis subspaces $V_{m}^{(k)}$ are images of basis subspaces $\tilde{V}_{m}^{(k)}$ under operator $T_{N}$, which is bounded and has bounded inverse for $N$ large enough (see proof of Theorem 2.1). Thus operator $T_{N}$ transforms Riesz basis to another Riesz basis, but of course the choice of $N$ depends on $\mathcal{A}$. Hence the constants can be chosen as $C_{A}=c_{A}^{-1}=\left\|T_{N}\right\|^{2}\left\|T_{N}^{-1}\right\|^{2}\|\tilde{T}\|^{2}\left\|\tilde{T}^{-1}\right\|^{2}$, where the operator $\tilde{T}$ transforms Riesz basis from subspaces $\tilde{V}_{k}$ onto orthonormal basis.

Space $M_{2}^{(N)}$ is the direct sum of $Q_{m}^{(N)}, m=1,2, \ldots, \ell$, where $Q_{m}^{(N)}=\overline{\bigoplus_{|k|>N} V_{m}^{(k)}}$. For any $x \in M_{2}^{(N)}$, using the above and (24), we have

$$
\left\|e^{\mathcal{A} t} x\right\|^{2} \leq\left\|\sum_{m=1}^{\ell} \sum_{|k|>N} e^{\mathcal{A} t} x_{m}^{(k)}\right\|^{2} \leq C_{A} \sum_{m=1}^{\ell} \sum_{|k|>N}\left\|e^{A_{m}^{(k)} t} x_{m}^{(k)}\right\|^{2} .
$$

Then

$$
\left\|e^{\mathcal{A} t} x\right\|^{2} \leq C_{A} \sum_{m=1}^{\ell} e^{2 \omega_{m} t} \sum_{|k|>N}\left\|e^{A_{m}^{(k), 0} t} x_{m}^{(k)}\right\|^{2},
$$

where $\omega_{m}=\sup \left\{\operatorname{Re} \lambda: \lambda \in \sigma\left(A_{m}^{(k)}\right),|k|>N\right\}$ and $A_{m}^{(k), 0}=A_{m}^{(k)}-\tilde{\lambda}_{m}^{(k)} I$. Substituting $x_{m}^{(k)}=T_{N}^{-1} \tilde{x}_{m}^{(k)}$ we obtain

$$
\left\|e^{\mathcal{A} t} x\right\|^{2} \leq C_{A}\left\|T_{N}^{-1}\right\|^{2} \sum_{m=1}^{\ell} e^{2 \omega_{m} t} \sum_{|k|>N}\left\|e^{B_{m}^{(k)} t} \tilde{x}_{m}^{(k)}\right\|^{2}
$$


where $\mathcal{B}_{m}^{(k)}$ is defined by $\mathcal{B}_{m}^{(k)}=T_{N} A_{m}^{(k), 0} T_{N}^{-1}$. For any fixed $\varepsilon$ and $A_{2}, A_{3}$ there exists $N$ large enough (see Lemma 2.2) that $\left\|B_{m}^{(k)}-\tilde{A}_{m}^{(k), 0}\right\|<\varepsilon$. Thus from Lemma 2.3 we obtain that there exists constant $M$, which is independent of $\mathcal{A}$, such that

$$
\left\|e^{B_{m}^{(k)} t} \tilde{x}_{m}^{(k)}\right\| \leq M\left(t^{p_{m}-1}+1\right)\left\|\tilde{x}_{m}^{(k)}\right\| .
$$

Hence (25) can be rewritten in the form

$$
\left\|e^{\mathcal{A} t} x\right\|^{2} \leq C_{A}\left\|T_{N}^{-1}\right\|^{2} M^{2} \sum_{m=1}^{\ell} e^{2 \omega_{m} t}\left(t^{p_{m}-1}+1\right)^{2} \sum_{|k|>N}\left\|\tilde{x}_{m}^{(k)}\right\|^{2} .
$$

For each $m>m_{0}$ there exists constant $M_{0}$ such that $e^{\omega_{m} t}\left(t^{p_{m}-1}+1\right) \leq M_{0} e^{\omega t}, t \geq 0$, $m>m_{0}$. Using the fact that $C_{A}=1 / c_{A}, M_{0} \geq 1$ and $\|\tilde{x}\| \leq\left\|T_{N}\right\| \cdot\|x\|$ we obtain

$$
\left\|e^{\mathcal{A} t} x\right\|^{2} \leq\left(C_{A} M M_{0}\left\|T_{N}\right\|\left\|T_{N}^{-1}\right\|\right)^{2} e^{2 \omega t}\left(t^{p-1}+1\right)^{2}\|x\|^{2} .
$$

Hence there exists constant $M_{p}:=C_{A} M M_{0}\left\|T_{N}\right\|\left\|T_{N}^{-1}\right\|$, which is independent of $A_{2}, A_{3}$ such that for some large enough $N$,

$$
\left\|e^{\mathcal{A} t} x\right\| \leq M_{p} e^{\omega t}\left(t^{p-1}+1\right)^{2}\|x\|, \quad x \in M_{2}^{(N)} .
$$

To prove part (ii) one needs to check that all constants used in proof of Theorem 2.1(ii) can be chosen independently on $A_{2}, A_{3}$ for some $N$ large enough. In particular constant $M_{T}$ is determined by the constant $M_{\delta}$, which depends only on dimension $n$ and $\delta$ (see Lemma 2.3). For any $A_{2}, A_{3}$ we can choose $N$ large enough such that $1 / 2<\left\|T_{N}\right\|,\left\|T_{N}^{-1}\right\|<3 / 2$ (see proof of Theorem 2.1(i)). The rest of the proof is the same as in Theorem 2.1.

\section{Maximal Asymptotics and Estimation of Individual Solutions Growth}

Knowing the asymptotic behaviour of $\left\|e^{\mathcal{A} t}\right\|$ one can analyse the behaviour of individuals $\left\|e^{\mathcal{A} t} x\right\|$. The question about existence of the solution of the fastest growth is of particular interest. This problem was studied for an abstract linear differential equation in Banach space [17]. We say that equation $\dot{x}=A x$ (or semigroup $e^{A t}, t \geq$ 0 ) has maximal asymptotics if there exists a real, positive function $f(t), t \geq 0$, such that the function $\frac{\left\|e^{A t}\right\|}{f(t)}$ is bounded on $[a,+\infty)$, for some $a \geq 0$, and there exists at least one $x_{0} \in X$ such that

$$
\lim _{t \rightarrow+\infty} \frac{\left\|e^{A t} x_{0}\right\|}{f(t)}=1 .
$$

It is easy to see that every finite-dimensional system has maximal asymtotics. Thus the question about maximal asymptotics is interesting only in the case of infinitedimensional systems. In particular, it was shown (see Theorem 5, 9 [17]) that if $\rho(A) \cap\left\{\lambda: \operatorname{Re} \lambda=\omega_{0}\right\}$ is at most countable, and the adjoint operator $A^{*}$ does not possess eigenvalues with real part $\omega_{0}$ then the equation $\dot{x}=A x$ does not have any 
maximal asymptotics. If, in addition, function $\log f(t)$ concaves down then the function $f$ increases faster then any solution of the system $\dot{x}=A x$, i.e.

$$
\lim _{t \rightarrow+\infty} \frac{\left\|e^{A t} x\right\|}{f(t)}=0
$$

for any $x \in X$. As a consequence of the above and of previous section results we obtain the following.

Theorem 3.1 Given operator $\mathcal{A}$ from Eq. (2), and eigenvalues $\lambda \in \sigma(\mathcal{A})$ satisfying $\operatorname{Re} \lambda<\omega$, with $\omega=\sup _{\lambda \in \sigma(\mathcal{A})} \operatorname{Re} \lambda$, we have

$$
\lim _{t \rightarrow \infty} \frac{\left\|e^{\mathcal{A} t} x\right\|}{M_{p} e^{\omega t} t^{p-1}}=0
$$

for any initial state $x \in M_{2}$. If, in addition, the matrix $A_{-1}$ has only one Jordan block corresponding to maximal (modulus) eigenvalue, then for any positive function $g(t)$, with $\frac{g(t)}{M_{p} e^{\omega t}\left(t^{p-1}\right)} \rightarrow 0$ as $t \rightarrow+\infty$, there exists an initial state $x \in M_{2}$ such that

$$
\frac{\left\|e^{\mathcal{A} t} x\right\|}{g(t)} \text { is unbounded on the interval }[0,+\infty)
$$

Proof The function $M_{p} e^{\omega t}\left(t^{p-1}+1\right)$ is the upper bound of $\left\|e^{\mathcal{A} t}\right\|$ and logarithm of $M_{p} e^{\omega t}\left(t^{p-1}+1\right)$ concaves down. Therefore (26) follows directly from Theorem 9 [17]. Now let us assume that matrix $A_{-1}$ has only one Jordan block corresponding to maximal (modulus) eigenvalue (i.e. $p=q$ ) and for any initial state $x \in M_{2}$ the quotient $\frac{\left\|e^{\mathcal{A} t} x\right\|}{g(t)}$ is bounded on the interval $[0,+\infty)$. From Banach-Steinhaus Theorem we obtain that the operator $e^{\mathcal{A} t} / g(t)$ is bounded by some constant $M$. But by Theorem $2.1\left\|e^{\mathcal{A} t}\right\| \geq m M_{p} e^{\omega t}\left(t^{p-1}\right)$, which means

$$
m \frac{M_{p} e^{\omega t}\left(t^{p-1}\right)}{g(t)} \leq \frac{\left\|e^{\mathcal{A} t}\right\|}{g(t)} \leq M
$$

and that contradicts the fact that $\frac{M_{p} e^{\omega t}\left(t^{p-1}\right)}{g(t)}$ tends to infinity.

In the following theorems we describe the conditions under which the Eq. (2) does or does not have maximal asymptotics. We recall that $\omega=\sup \{\operatorname{Re} \lambda: \lambda \in \sigma(\mathcal{A})\}$, $\tilde{\omega}=\sup \{\operatorname{Re} \lambda: \lambda \in \sigma(\tilde{\mathcal{A}})\}$, and we denote by $q_{0}$ the maximal order of rootvector corresponding to eigenvalue $\lambda \in \sigma(\mathcal{A})$ with $\operatorname{Re} \lambda=\omega$. If $\{\lambda \in \sigma(\mathcal{A}): \operatorname{Re} \lambda=\omega\}=\varnothing$ then we put $q_{0}:=0$. We notice that in general $\omega \geq \tilde{\omega}$ and the following theorem describes asymptotic behaviour of Eq. (2) in both cases $\omega>\tilde{\omega}$ and $\omega=\tilde{\omega}$.

Theorem 3.2 If a real, positive function $f$ is maximal asymptotics of Eq. (2) then $q_{0} \geq 1$ and $f \approx t^{q_{0}-1} e^{\omega t}$ i.e. there exist positive constants $c, C$ with

$$
0<c=\liminf _{t \rightarrow+\infty} \frac{t^{q_{0}-1} e^{\omega t}}{f} \leq \limsup _{t \rightarrow+\infty} \frac{t^{q_{0}-1} e^{\omega t}}{f}=C<+\infty .
$$


Moreover, function $t^{q_{0}-1} e^{\omega t}$ is also maximal asymptotics of this equation.

Proof If there is no eigenvalue of $\mathcal{A}$ on the line $\operatorname{Re} z=\omega$ (i.e. $q_{0}=0$ ), then Eq. (2) can not possess any maximal asymptotics (see [17] for more details). Therefore $q_{0} \geq 1$. Now we prove that $C<+\infty$. We denote by $x_{0}$ the rootvector of operator $\mathcal{A}$ of order $q_{0}$ corresponding to an eigenvalue of real part $\omega$. The solution corresponding to the state $x_{0}$ satisfies the following inequality

$$
\frac{\left\|e^{\mathcal{A} t} x_{0}\right\|}{t^{q_{0}-1} e^{\omega t}} \geq m, \quad t \geq t_{0},
$$

where $m, t_{0}$ are some positive constants. Function $f$ is maximal asymptotics, what means, in particular, that there exist constants $M, t_{0}$ such that for all $x \in M_{2}$

$$
\frac{\left\|e^{\mathcal{A} t} x\right\|}{f(t)} \leq M\|x\|, \quad t \geq t_{0} .
$$

Putting $x=x_{0}$ into (28) and combining with (27) we get

$$
\frac{t^{q_{0}-1} e^{\omega t}}{f(t)} \leq \frac{M\left\|x_{0}\right\|}{m} \quad \text { for } t \geq t_{0}
$$

and that proves the first part of the theorem.

To prove that $c>0$, we proceed by contradiction. Let us assume that $c=0$. This implies that there exists a sequence $t_{j} \rightarrow+\infty$ such that

$$
\lim _{j \rightarrow+\infty} \frac{t_{j}^{q_{0}-1} e^{\omega t_{j}}}{f\left(t_{j}\right)}=0 .
$$

Now, for any $x \in M_{2}$ we have

$$
\lim _{j \rightarrow+\infty} \frac{\left\|e^{\mathcal{A} t_{j}} x\right\|}{f\left(t_{j}\right)}=0 .
$$

Indeed, to see this, we observe first, that if the state $x \in V_{m}^{(k)}$, for $k \in \mathbb{Z}, m=1, \ldots, \ell$, then (29) follows from the fact that every subspace $V_{m}^{(k)}$ is finite-dimensional and for each $m, k$ there exist constants $M_{m, k}, t_{m, k}$ such that $\left\|e^{\mathcal{A} t} x\right\| \leq M_{m, k} t^{q_{0}-1} e^{\omega t}\|x\|$, $x \in V_{m}^{(k)}, t>t_{m, k}$. Hence

$$
0 \leq \frac{\left\|e^{\mathcal{A} t_{j}} x\right\|}{f\left(t_{j}\right)} \leq M_{m, k}\|x\| \cdot \frac{t_{j}^{q_{0}-1} e^{\omega t_{j}}}{f\left(t_{j}\right)} \stackrel{j \rightarrow+\infty}{\longrightarrow} 0 .
$$

If $x$ is a finite combination of states from $V_{m}^{(k)}$ then (29) follows for the same reason. Let now $x \in \overline{\bigoplus_{k \in \mathbb{Z} ; m=1, \ldots, \ell} V_{m}^{(k)}}$. We take sequence $\left\{x_{n}\right\} \subset \bigoplus_{k \in \mathbb{Z} ; m=1, \ldots, \ell} V_{m}^{(k)}$, such that $x_{n} \rightarrow x$. Using triangle inequality and (28) we obtain

$$
0 \leq \frac{\left\|e^{\mathcal{A} t} x\right\|}{f(t)} \leq M\left\|x-x_{n}\right\|+\frac{\left\|e^{\mathcal{A} t} x_{n}\right\|}{f(t)}, \quad t \geq t_{0} .
$$


Taking $t=t_{j}$ and letting $j$ tend to $+\infty$ in the above, we obtain for every $n \in \mathbb{N}$

$$
0 \leq \lim _{j \rightarrow+\infty} \frac{\left\|e^{\mathcal{A} t_{j}} x\right\|}{f\left(t_{j}\right)} \leq M\left\|x-x_{n}\right\|+0 .
$$

Then we apply the limit with $n \rightarrow+\infty$ in the above inequality to get

$$
\lim _{j \rightarrow+\infty} \frac{\left\|e^{\mathcal{A} t_{j}} x\right\|}{f\left(t_{j}\right)}=0, \quad \text { for any } x \in \overline{\bigoplus_{k \in \mathbb{Z} ; m=1, \ldots, \ell} V_{m}^{(k)}}=M_{2} .
$$

But since the function $f$ is maximal asymptotics, then there exists a state $x_{0} \in M_{2}$, such that

$$
\lim _{j \rightarrow+\infty} \frac{\left\|e^{\mathcal{A} t_{j}} x_{0}\right\|}{f\left(t_{j}\right)}=1,
$$

a contradiction. Finally, we observe that the proved inequality implies boundedness of the function

$$
\varphi(t)=\frac{\left\|e^{\mathcal{A} t} x\right\|}{t^{q_{0}-1} e^{\omega t}}, \quad t \geq a,
$$

for all $x \in M_{2}$. Besides, for some $m_{0}, k_{0}$, there exists a rootvector $x_{0} \in V_{m_{0}}^{\left(k_{0}\right)}$ such that

$$
\lim _{t \rightarrow+\infty} \frac{\left\|e^{\mathcal{A} t} x_{0}\right\|}{t^{q_{0}-1} e^{\omega t}}=1 .
$$

Thus the function $t^{q_{0}-1} e^{\omega t}$ is also maximal asymptotics.

Theorem 3.3 The following hold.

(i) If $\omega>\tilde{\omega}$ then Eq. (2) posses the maximal asymptotics, given by $t^{q_{0}-1} e^{\omega t}$.

(ii) If $\omega=\tilde{\omega}$ then the existence of maximal asymptotics depends on relation between parameters $q, q_{0}, p$. (1) if $q_{0}<q$ then Eq. (2) does not posses any maximal asymptotics, (2) if $q \leq q_{0}<p$ then Eq. (2) may posses and may not posses the maximal asymptotics, (3) if $q_{0} \geq p$ then Eq. (2) posses the maximal asymptotics, which is $t^{q_{0}-1} e^{\omega t}$.

Proof (i) If there exists $\lambda \in \sigma(\mathcal{A}), \operatorname{Re} \lambda \geq \tilde{\omega}$, then for at least one eigenvalue $\lambda_{0} \in \sigma(\mathcal{A})$ we have $\operatorname{Re} \lambda_{0}=\max \{\operatorname{Re} \lambda, \lambda \in \sigma(\mathcal{A})\}$ and the size of Jordan block corresponding to $\lambda_{0}$ equals $q_{0}$. There is only finite number of eigenvalues $\lambda \in$ $\sigma(\mathcal{A})$ with $\operatorname{Re} \lambda_{0}-\operatorname{Re} \lambda<\frac{1}{2}(\omega-\tilde{\omega})$ because the radii $r_{k}$ of circles centered at $\tilde{\omega}+i\left(\arg \mu_{0}+2 k \pi\right)$ countaining the eigenvalues of operator $\mathcal{A}$ tend to 0 as $k \rightarrow \infty$. Thus $\left\|e^{\mathcal{A} t}\right\|=O\left(t^{q_{0}-1} e^{\lambda_{0} t}\right)$. On the other hand there is rootvector $x_{0}$ corresponding to $\lambda_{0}$ such that

$$
\lim _{t \rightarrow \infty} \frac{\left\|e^{\mathcal{A} t} x_{0}\right\|}{t^{q_{0}-1} e^{\omega t}}=1 .
$$

This means that the function $t^{q_{0}-1} e^{\omega t}$ is maximal asymptotics. 
(ii) Let $q_{0}<q$. We argue by contradiction. Let $f(t)$ be maximal asymptotics. According to Theorem 3.2 we have $f \approx t^{q_{0}-1} e^{\omega t}$. On the other hand it is shown (Remark 3 [17]) that $f \approx\left\|e^{\mathcal{A} t}\right\|$. From Theorem 2.1 we have also $\left\|e^{\mathcal{A} t}\right\| \geq M t^{q-1} e^{\omega t}$. This implies that $q=q_{0}$, a contradiction. Let $q_{0} \geq p$. It is easy to see that the function $f(t)=e^{\omega t} t^{q_{0}-1}$ is maximal asymptotics. Indeed from Corollary 2.4 for each $x \in M_{2}$ we have $\frac{\left\|e^{\mathcal{A} t} x\right\|}{f(t)} \leq M\|x\|$ and there exists a rootvector $x_{0}$ of order $q_{0}$ such that $\frac{\left\|e^{\mathcal{A} t} x_{0}\right\|}{f(t)} \rightarrow 1$.

If $q_{0} \geq p$ then it is clear that there is a rootvector $x_{0}$ corresponding to $\lambda_{0}$ such that

$$
\lim _{t \rightarrow \infty} \frac{\left\|e^{\mathcal{A} t} x_{0}\right\|}{t^{q_{0}-1} e^{\omega t}}=1
$$

The inequality

$$
\frac{\left\|e^{\mathcal{A} t}\right\|}{t^{q_{0}-1} e^{\omega t}} \leq M
$$

follows directly from the Corollary 2.4. Thus the function $t^{q_{0}-1} e^{\omega t}$ is a maximal asymptotics.

If $q \leq q_{0}<p$ then we do not know the exact behaviour of $\left\|e^{\mathcal{A} t}\right\|$. As we see (Example 2.1 and Example 2.2) the function $\varphi(t)=\left\|e^{\mathcal{A} t}\right\|$ may behave asymptotically like $t^{q_{0}-1} e^{\omega t}$ or $t^{p-1} e^{\omega t}$. According to Theorem 3.2 in the first case the function $t^{q_{0}-1} e^{\omega t}$ is maximal asymptotics, in the second case function $t^{q_{0}-1} e^{\omega t}$ is the best candidate for being maximal asymptotics but it is easy to see that

$$
\frac{\left\|e^{\mathcal{A} t}\right\|}{t^{q_{0}-1} e^{\omega t}}
$$

is not bounded and hence Eq. (2) does not have any maximal asymptotics.

\section{Selected Applications for Control Systems}

In this section we give some applications of our results for control systems. Namely, we consider a controlled neutral type system of the form

$$
\dot{z}(t)=A_{-1} \dot{z}(t-1)+\int_{-1}^{0} A_{2}(\theta) \dot{z}(t+\theta) d \theta+\int_{-1}^{0} A_{3}(\theta) z(t+\theta) d \theta+B u,
$$

where $B$ is $n \times r$ matrix. Such system can be rewritten in the operator form

$$
\dot{x}=\mathcal{A} x+\mathcal{B} u, \quad x \in D(\mathcal{A}) \subset M_{2},
$$

where $\mathcal{A}$ is introduced above and $\mathcal{B}=\left(\begin{array}{l}B \\ 0\end{array}\right)$. We study two problems. First we consider the closed-loop control for system (31) with a feedback law of the form

$$
u(t)=\mathcal{F}\left(\begin{array}{c}
y \\
z_{t}(\cdot)
\end{array}\right)=\int_{-1}^{0} F_{2}(\theta) \dot{z}(t+\theta) d \theta+\int_{-1}^{0} F_{3}(\theta) z(t+\theta) d \theta,
$$


where $F_{2}(\cdot), F_{3}(\cdot) \in L_{2}\left(-1,0 ; \mathbb{C}^{r \times n}\right)$. We want to investigate how adding of a control of this type can affect the system. In particular, we check the asymptotic behaviour of solutions under such controls. Next we consider the case of programming controls satisfying the constraint $\|u(t)\| \leq 1$. Our goal is to describe the asymptotic behaviour of the diameters of the sets of null-reachable states.

Let us discuss the first question in detail. It was shown in [16] that for any linear feedback control of the form (32) the displaced eigenvalues of operator $\mathcal{A}+\mathcal{B F}$ are still in the discs $L_{m}^{(k)}$ centered in $\tilde{\lambda}_{m}^{(k)}$, for $|k|$ large enough. On the other hand for any choice of small enough displacements there is a feedback, which dislocates eigenvalues to desired points under the condition that all eigenvalues $\tilde{\lambda}_{m}^{(k)}$ are simple. This means that for any choice of sequences $\tau_{m}^{(k)} \in L_{m}^{(k)}, k \in \mathbb{Z}, m=1, \ldots, \ell$ there exists a linear feedback $\mathcal{F}$ of the form (32) such that $\lambda_{m}^{(k)}=\tau_{m}^{(k)}$. The growth of the norm of the semigroup corresponding to Eq. (31) under no control is described by Corollary 2.4 as

$$
m e^{\omega t} t^{\max \left\{q, q_{0}\right\}-1} \leq\left\|e^{\mathcal{A} t}\right\| \leq M e^{\omega t} t^{\max \left\{p, q_{0}\right\}-1}, \quad t>1 .
$$

The following Statement describes how the behaviour of solutions can be changed under a closed-loop control (32).

Statement 4.1 Let us consider a control system (31). If there exists a feedback $\mathcal{F}$ of the form (32), which displaces the spectrum of operator $\mathcal{A}$ in such a way that $\operatorname{Re} \lambda<\tilde{\omega}$ for all $\lambda \in \sigma(\mathcal{A}+\mathcal{B} \mathcal{F})$, then for some positive constants $m, M$ we have

$$
m e^{\tilde{\omega} t} t^{q-1} \leq\left\|e^{(\mathcal{A}+\mathcal{B} \mathcal{F}) t}\right\| \leq M e^{\tilde{\omega} t} t^{p-1}, \quad t>1 .
$$

In particular this means that no choice of a control of the form (32) can decrease the exponents $\tilde{\omega}, q$ or increase $p$.

Note that for a simple $\tilde{\lambda}: \operatorname{Re} \tilde{\lambda}=\tilde{\omega}$ the assumption of Statement 4.1 is satisfied.

Proof Assertion follows from the fact that the system (31)-(32) can be represented as

$$
\dot{x}=\mathcal{A}_{1} x, \quad x \in D\left(\mathcal{A}_{1}\right) \subset M_{2},
$$

where $\mathcal{A}_{1}=\mathcal{A}+\mathcal{B} \mathcal{F}, D\left(\mathcal{A}_{1}\right)=D(\mathcal{A})$. Operator $\mathcal{A}_{1}$ generates a $C_{0}$-semigroup and also is of the form (3) with the same matrix $A_{-1}$ and disturbed $A_{2}, A_{3}$ (see [16] for more details). Thus assertion follows directly from Theorem 2.1.

Consider now the problem of null-controllability for the system (31). The detailed analysis of this problem for the case of unrestricted $u$ is given in the work [14]. In particular it was proven that for sufficiently large time $T$ if some special conditions on the matrices of system (30) are satisfied then system (31) is exact controllable (see Theorem 1.2 [14] for more details). The latter means that the null-reachability set is maximal possible, i.e.

$$
\mathcal{R}_{T}=\left\{\int_{0}^{T} e^{\mathcal{A} t} \mathcal{B} u(t) d t, u \in L_{2}\left([0, T], \mathbb{C}^{r}\right)\right\}=D(\mathcal{A}) .
$$


Let us consider the case of a bounded control: $\|u(t)\| \leq 1$. In this case nullreachability set $\tilde{\mathcal{R}}_{T}$ is given by

$$
\tilde{\mathcal{R}}_{T}=\left\{\int_{0}^{T} e^{\mathcal{A} t} \mathcal{B} u(t) d t, u \in L_{2}\left([0, T], \mathbb{C}^{r}\right),\|u(t)\|_{\mathbb{C}^{r}} \leq 1\right\} \subset D(\mathcal{A}) .
$$

Our goal is to find the asymptotical growth of the diameter of set $\tilde{\mathcal{R}}_{T}$ in time. Since $\tilde{\mathcal{R}}_{T} \subset D(\mathcal{A})$ the natural norm for our estimation is

$$
\|x\|_{D(\mathcal{A})}=\|\mathcal{A} x\|_{M_{2}}+\|x\|_{M_{2}} .
$$

First we prove the following lemma.

Lemma 4.2 Let $T=1$, then there exists a constant $C$ - determined by the parameters of system (30)-such that for any $x \in \tilde{\mathcal{R}}_{1}$

$$
\|x\|_{D(\mathcal{A})} \leq C .
$$

Proof Let $x \in \tilde{\mathcal{R}}_{1}$, we have

$$
\|x\|_{D(\mathcal{A})}^{2}=\left\|\int_{0}^{1} e^{\mathcal{A} t} \sum_{j=1}^{r} b_{j} u_{j}(t) d t\right\|_{D(\mathcal{A})}^{2} \leq \sum_{j=1}^{r}\left\|\int_{0}^{1} e^{\mathcal{A} t} b_{j} u_{j}(t) d t\right\|_{D(\mathcal{A})}^{2},
$$

where $b_{j}, j=1, \ldots, r$ are the columns of operator $\mathcal{B}$. Since the family $\left\{V_{m}^{(k)}:|k|>\right.$ $N, k \in \mathbb{Z}\} \cup\left\{W_{N}\right\}$ constitutes Riesz basis in $M_{2}$, then

$$
\|x\|_{D(\mathcal{A})}^{2} \leq C_{1} \sum_{m=1}^{\ell} \sum_{|k|>N} k^{2}\left\|P_{m}^{(k)} \int_{0}^{1} e^{\mathcal{A} t} \sum_{j=1}^{r} b_{j} u_{j}(t) d t\right\|_{M_{2}}^{2},
$$

where $C_{1}>0$ is a constant. Without loss of generality we assume that $r=\ell=1$ and omit indices $j, m$,

$$
\begin{aligned}
& \|x\|_{D(\mathcal{A})}^{2} \\
& \quad \leq C_{2} \sum_{|k| \geq N} k^{2}\left(\left\|\int_{0}^{1}\left(P_{k} e^{\mathcal{A} t}-\tilde{P}_{k} e^{\tilde{\mathcal{A}} t}\right) b u(t) d t\right\|_{M_{2}}^{2}+\left\|\int_{0}^{1} \tilde{P}_{k} e^{\tilde{\mathcal{A}} t} b u(t) d t\right\|_{M_{2}}^{2}\right) .
\end{aligned}
$$

We estimate both integrals using the properties of operators $\tilde{P}_{k}, P_{k}, S_{k}, A_{0}$ defined and discussed in detail in Sect. 2. Riesz projectors $\tilde{P}_{k}$ restricted to subspaces $V_{k}$ are close to identity, hence there exists a constant $m_{1}$ such that $\left\|\tilde{P}_{k} x\right\| \geq m_{1}\|x\|$ for any $x \in V_{k}$ and $|k|$ large enough. Applying this fact and Lemma 3.1 [14], which states that there exists a sequence $\left\{\alpha_{k}\right\} \in \ell_{2}$ such that $\left\|\tilde{P}_{k}\left(P_{k} e^{\mathcal{A} t}-\tilde{P}_{k} e^{\tilde{\mathcal{A}} t}\right) b u(t)\right\|_{M_{2}} \leq \frac{\alpha_{k}}{|k|}$ we can estimate the first integral as follows

$$
\|x\|_{D(\mathcal{A})}^{2} \leq C_{3}+C_{2} \sum_{|k| \geq N} k^{2}\left\|\int_{0}^{1} \tilde{P}_{k} e^{\tilde{\mathcal{A}} t} b u(t) d t\right\|_{M_{2}}^{2},
$$


where $C_{3}$ is a new constant. Next we use $\tilde{P}_{k} e^{\tilde{\mathcal{A}} t}=e^{\tilde{\lambda}_{k} t} S_{k}^{-1} e^{A_{0} t} S_{k}$ and the fact that the norms of $S_{k}, S_{k}^{-1}$ are uniformly bounded, thus

$$
\|x\|_{D(\mathcal{A})}^{2} \leq C_{3}+C_{4} \sum_{|k| \geq N} k^{2}\left\|\int_{0}^{1} e^{\tilde{\lambda}_{k} t} e^{A_{0} t} S_{k} \tilde{P}_{k} b u(t) d t\right\|_{M_{2}}^{2} .
$$

Since $\tilde{\lambda}_{k}=\operatorname{Re} \tilde{\lambda}_{1}+i(2 k \pi+c)$, where $c$ is some constant, we have

$$
\|x\|_{D(\mathcal{A})}^{2} \leq C_{3}+C_{5} \sum_{|k| \geq N} k^{2}\left|\int_{0}^{1} e^{i 2 k \pi t} e^{\operatorname{Re} \tilde{\lambda}_{1} t} e^{A_{0} t} u(t) d t\right|^{2}\left\|\tilde{P}_{k} b\right\|_{M_{2}}^{2} .
$$

Applying Lemma 3.2 [14], which states that $\left\|\tilde{P}_{k} b\right\| \leq \frac{\alpha}{|k|}$, for some constant $\alpha$ and any $k$, we get

$$
\|x\|_{D(\mathcal{A})}^{2} \leq C_{3}+C_{5} \sum_{|k| \geq N}\left|\left\langle e^{\operatorname{Re} \tilde{\lambda}_{1} \cdot} e^{A_{0} \cdot} u(\cdot), e^{i 2 k \pi \cdot}\right\rangle_{L^{2}(0,1)}\right|^{2} .
$$

The last series converges and its sum is at most $\left\|e^{\operatorname{Re} \tilde{\lambda}_{1} \cdot} e^{A_{0} \cdot} u(\cdot)\right\|_{L^{2}(0,1)}^{2}$. Thus under our assumption that $\|u(t)\| \leq 1$, we conclude that there exists a constant $C$ such that $\|x\|_{D(\mathcal{A})}^{2} \leq C$.

The result obtained above allows us to describe asymptotic behaviour of diameter of the set $\tilde{\mathcal{R}}_{T}$. To this end we take $T=n$ for $n \in \mathbb{N}$ and estimate diam $\tilde{\mathcal{R}}_{n}$. Since set $\tilde{\mathcal{R}}_{n}$ is symmetric, $\operatorname{diam} \tilde{\mathcal{R}}_{n}=2 \sup \left\{\|x\|: x \in \tilde{\mathcal{R}}_{n}\right\}$. The elements of $\tilde{\mathcal{R}}_{n}$ are of the form $\int_{0}^{n} e^{\mathcal{A} t} \mathcal{B} u(t) d t$, hence we can split this integral as $\sum_{k=0}^{n-1} \int_{k}^{(k+1)} e^{\mathcal{A} t} \mathcal{B} u(t) d t$ and substitute $s=t-k$. This way we obtain

$$
\operatorname{diam} \tilde{\mathcal{R}}_{n} \leq \operatorname{diam} \tilde{\mathcal{R}}_{1} \sum_{k=0}^{n-1}\left\|e^{\mathcal{A} k}\right\|_{M_{2}}
$$

The last norm can be estimated using Theorem 2.1, thus

$$
\operatorname{diam} \tilde{\mathcal{R}}_{n} \leq \operatorname{diam} \tilde{\mathcal{R}}_{1} \sum_{k=0}^{n-1} e^{\omega k} k^{p-1}
$$

Lemma 4.2 and (35) imply the following

Statement 4.3 The asymptotics of $\tilde{\mathcal{R}}_{T}$ is as follows: if $\omega<0$ then there exists a positive constant $M$ such that

$$
\operatorname{diam} \tilde{\mathcal{R}}_{T} \rightarrow M, \quad T \rightarrow+\infty
$$

if $\omega=0$ then there exists a positive constant $M$ such that

$$
\frac{\operatorname{diam} \tilde{\mathcal{R}}_{T}}{T^{p}} \rightarrow M, \quad T \rightarrow+\infty
$$


if $\omega>0$ then there exists a positive constant $M$ such that

$$
\frac{\operatorname{diam} \tilde{\mathcal{R}}_{T}}{e^{\omega T} T^{p-1}} \rightarrow M, \quad T \rightarrow+\infty .
$$

Open Access This article is distributed under the terms of the Creative Commons Attribution License which permits any use, distribution, and reproduction in any medium, provided the original author(s) and the source are credited.

\section{Appendix}

In this section we recall some properties of divided differences and the statements on determinants used in the proof of Lemma 2.3.

Property 1 Let $f: \mathbb{C} \rightarrow \mathbb{C}$ be an analytic function, then divided difference has a representation as a series of the form

$$
f\left[x_{0}, x_{1}, \ldots, x_{m}\right]=\sum_{n=m}^{\infty} \frac{f^{(n)}(0)}{n !} \sum_{i_{0}+\cdots+i_{m}=n-m} x_{0}^{i_{0}} x_{1}^{i_{1}} \ldots x_{m}^{i_{m}} .
$$

Corollary 1 Divided difference is symmetric, that is

$$
f\left[x_{0}, x_{1}, \ldots, x_{n}\right]=f\left[x_{\sigma(0)}, x_{\sigma(1)}, \ldots, x_{\sigma(n)}\right] \text { for any permutation } \sigma .
$$

Corollary 2 Divided difference $f\left[x_{0}, x_{1}, \ldots, x_{n}\right]$ can be extended to the whole $\mathbb{C}^{n+1}$ by the formula (36).

Property 2 Let us denote $\{x \in \mathbb{C}: \operatorname{Re} x \leq 0\}$ by $\mathbb{C}^{-}$. If $f(x)=e^{x}$ then its divided difference is bounded on $\left(\mathbb{C}^{-}\right)^{n+1}$, i.e. for any $n$ there exists constant $M_{n}>0$, such that $\left|f\left[x_{0}, x_{1}, \ldots, x_{n}\right]\right| \leq M_{n}$ for all $x \in\left(\mathbb{C}^{-}\right)^{n+1}$.

Property 3 For any $\lambda=\left(\lambda_{1}, \ldots, \lambda_{n}\right) \in\left(\mathbb{C}^{-}\right)^{n}$, there exists constant $M(n, \delta)$ independent of $\lambda, t$, such that for all $t \geq 1$

$$
\left|f\left[\lambda_{1} t, \lambda_{2} t, \ldots, \lambda_{n} t\right]\right| \leq \frac{M(n, \delta)}{t^{n-p}}, \quad t \geq 1 .
$$

Property 4 If $f(x)=\exp (x), x \in \mathbb{C}$, then for any $z \in \mathbb{C}$ and $n \in \mathbb{N}$ we have

$$
f\left[x_{0}, x_{1}, \ldots, x_{n}\right]=\exp (z) f\left[x_{0}-z, x_{1}-z, \ldots, x_{n}-z\right] .
$$

Statement 1 If matrix $A \in M_{n}(\mathbb{C})$ has $n$ different eigenvalues $\lambda_{i}$ then the coefficients $\alpha_{k}(t)$ of the representation $e^{A t}=\sum_{k=0}^{n-1} \alpha_{k}(t) A^{k}$ can be written in the form $\alpha_{k}(t)=$ 
$\frac{W_{k}(t)}{W}$, where

$$
W_{k}(t)=\left|\begin{array}{cccc}
1 & 1 & \ldots & 1 \\
\lambda_{1} & \lambda_{2} & \ldots & \lambda_{n} \\
\vdots & \vdots & & \vdots \\
\lambda_{1}^{k-1} & \lambda_{2}^{k-1} & \ldots & \lambda_{n}^{k-1} \\
e^{\lambda_{1} t} & e^{\lambda_{2} t} & \ldots & e^{\lambda_{n} t} \\
\lambda_{1}^{k+1} & \lambda_{2}^{k+1} & \ldots & \lambda_{n}^{k+1} \\
\vdots & \vdots & & \vdots \\
\lambda_{1}^{n-1} & \lambda_{2}^{n-1} & \ldots & \lambda_{n}^{n-1}
\end{array}\right|, \quad W=\left|\begin{array}{ccccc}
1 & 1 & \ldots & 1 \\
\lambda_{1} & \lambda_{2} & \ldots & \lambda_{n} \\
\vdots & \vdots & \ddots & \vdots \\
\lambda_{1}^{n-1} & \lambda_{2}^{n-1} & \ldots & \lambda_{n}^{n-1}
\end{array}\right| .
$$

Proof Let $x_{i}, i=1, \ldots, n$ be the eigenvectors of matrix $A$ corresponding to eigenvalues $\lambda_{i}, i=1, \ldots, n$ respectively. So we have a system of $n$ equations

$$
e^{A t} x_{i}=\sum_{k=0}^{n-1} \alpha_{k}(t) A^{k} x_{i}, \quad i=1, \ldots, n
$$

Since $e^{A t} x_{i}=e^{\lambda_{i} t} x_{i}$ and $A^{k} x_{i}=\lambda_{i}^{k} x_{i}$, we have

$$
e^{\lambda_{i} t} x_{i}=\sum_{k=0}^{n-1} \alpha_{k}(t) \lambda_{i}^{k} x_{i}, \quad i=1, \ldots, n,
$$

which is equivalent to

$$
e^{\lambda_{i} t}=\sum_{k=0}^{n-1} \alpha_{k}(t) \lambda_{i}^{k}, \quad i=1, \ldots, n
$$

The above is a linear system with respect to $\alpha_{k}(t)$. Matrix of coefficients is a Vandermonde's matrix, with the determinant equal to $W$. Determinant $W$ is non zero because $\lambda_{i} \neq \lambda_{j}$ for $i \neq j$. Thus using Cramer's rule we obtain a solution $\alpha_{k}(t)=\frac{W_{k}(t)}{W}$. This concludes the proof. 
Statement 2 The quotient of determinants $\frac{W_{k}(t)}{W}$ can be written as

$\mid \begin{array}{ccccc}1 & 0 & 0 & \ldots & 0 \\ \lambda_{1} & 1 & 0 & \ldots & 0 \\ \lambda_{1}^{2} & \lambda_{2}+\lambda_{1} & 1 & \ldots & 0 \\ \vdots & \vdots & \vdots & & \vdots \\ \lambda_{1}^{k-1} & \sum_{i_{2}+i_{1}=k-2} \lambda_{2}^{i_{2}} \lambda_{1}^{i_{1}} & \sum_{i_{3}+i_{2}+i_{1}=k-3} \lambda_{3}^{i_{3}} \lambda_{2}^{i_{2}} \lambda_{1}^{i_{1}} & \ldots & 0 \\ f\left[\lambda_{1} t\right] & t f\left[\lambda_{2} t, \lambda_{1} t\right] & t^{2} f\left[\lambda_{3} t, \lambda_{2} t, \lambda_{1} t\right] & \ldots & t^{n-1} f\left[\lambda_{n} t, \ldots, \lambda_{1} t\right] \\ \lambda_{1}^{k+1} & \sum_{i_{2}+i_{1}=k} \lambda_{2}^{i_{2}} \lambda_{1}^{i_{1}} & \sum_{i_{3}+i_{2}+i_{1}=k-1} \lambda_{3}^{i_{3}} \lambda_{2}^{i_{2}} \lambda_{1}^{i_{1}} & \ldots & 0 \\ \vdots & \vdots & \vdots & & \vdots \\ \lambda_{1}^{n-1} & \sum_{i_{2}+i_{1}=n-2} \lambda_{2}^{i_{2}} \lambda_{1}^{i_{1}} & \sum_{i_{3}+i_{2}+i_{1}=n-3} \lambda_{2}^{i_{2}} \lambda_{2}^{i_{2}} \lambda_{1}^{i_{1}} & \ldots & 1\end{array}$

where $f(x)=e^{x}$.

Proof Let $g_{p}(x)=x^{p}$, we can rewrite determinant $W_{k}(t)$ as follows

$$
W_{k}(t)=\left|\begin{array}{cccc}
g_{0}\left(\lambda_{1}\right) & g_{0}\left(\lambda_{2}\right) & \ldots & g_{0}\left(\lambda_{n}\right) \\
g_{1}\left(\lambda_{1}\right) & g_{1}\left(\lambda_{2}\right) & \ldots & g_{1}\left(\lambda_{n}\right) \\
\vdots & \vdots & & \vdots \\
g_{k-1}\left(\lambda_{1}\right) & g_{k-1}\left(\lambda_{2}\right) & \ldots & g_{k-1}\left(\lambda_{n}\right) \\
f\left(\lambda_{1} t\right) & f\left(\lambda_{2} t\right) & \ldots & f\left(\lambda_{n} t\right) \\
g_{k+1}\left(\lambda_{1}\right) & g_{k+1}\left(\lambda_{2}\right) & \ldots & g_{k+1}\left(\lambda_{n}\right) \\
\vdots & \vdots & & \vdots \\
g_{n-1}\left(\lambda_{1}\right) & g_{n-1}\left(\lambda_{2}\right) & \ldots & g_{n-1}\left(\lambda_{n}\right)
\end{array}\right|
$$

Denote $j$-th column of $W_{k}(t)$ by $K_{j}$ and transform it in $n-1$ steps. First step: from the last column $K_{n}$ subtract the previous one, i.e. $K_{n-1}$, divide it by $\lambda_{n}-\lambda_{n-1}$ and put the same factor in front of determinant. We do the same with columns $K_{n-1}, \ldots, K_{2}$, i.e. we subtract the previous column and pull the corresponding difference of lambdas out of determinant. We denote it by $K_{j} \mapsto \frac{K_{j}-K_{j-1}}{\lambda_{j}-\lambda_{j-1}}$ for $j=n, n-1, \ldots, 2$. This operation does not change the value of the determinant. Using the definition of divided differences, we can rewrite $W_{k}$ as

$$
W_{k}(t)=\prod_{j=2}^{n}\left(\lambda_{j}-\lambda_{j-1}\right)\left|\begin{array}{cccc}
g_{0}\left[\lambda_{1}\right] & g_{0}\left[\lambda_{2}, \lambda_{1}\right] & \ldots & g_{0}\left[\lambda_{n}, \lambda_{n-1}\right] \\
g_{1}\left[\lambda_{1}\right] & g_{1}\left[\lambda_{2}, \lambda_{1}\right] & \ldots & g_{1}\left[\lambda_{n}, \lambda_{n-1}\right] \\
\vdots & \vdots & & \vdots \\
g_{k-1}\left[\lambda_{1}\right] & g_{k-1}\left[\lambda_{2}, \lambda_{1}\right] & \ldots & g_{k-1}\left[\lambda_{n}, \lambda_{n-1}\right] \\
f\left[\lambda_{1} t\right] & t f\left[\lambda_{2} t, \lambda_{1} t\right] & \ldots & t^{n-1} f\left[\lambda_{n} t, \lambda_{n-1} t\right] \\
g_{k+1}\left[\lambda_{1}\right] & g_{k+1}\left[\lambda_{2}, \lambda_{1}\right] & \ldots & g_{k+1}\left[\lambda_{n}, \lambda_{n-1}\right] \\
\vdots & \vdots & & \vdots \\
g_{n-1}\left[\lambda_{1}\right] & g_{n-1}\left[\lambda_{2}, \lambda_{1}\right] & \ldots & g_{n-1}\left[\lambda_{n}, \lambda_{n-1}\right]
\end{array}\right| .
$$


In $m$-th step we transform $K_{j} \mapsto \frac{K_{j}-K_{j-1}}{\lambda_{j}-\lambda_{j-m}}$ for $j=n, n-1, \ldots, 1+m$. After $n-1$ steps, we obtain $W_{k}(t)$ in the following form

$$
\begin{aligned}
& W_{k}(t) \\
& =\prod_{1 \leq i<j \leq n}\left(\lambda_{j}-\lambda_{i}\right)\left|\begin{array}{cccc}
g_{0}\left[\lambda_{1}\right] & g_{0}\left[\lambda_{2}, \lambda_{1}\right] & \ldots & g_{0}\left[\lambda_{n}, \lambda_{n-1}, \ldots, \lambda_{1}\right] \\
g_{1}\left[\lambda_{1}\right] & g_{1}\left[\lambda_{2}, \lambda_{1}\right] & \ldots & g_{1}\left[\lambda_{n}, \lambda_{n-1}, \ldots, \lambda_{1}\right] \\
\vdots & \vdots & & \vdots \\
g_{k-1}\left[\lambda_{1}\right] & g_{k-1}\left[\lambda_{2}, \lambda_{1}\right] & \ldots & g_{k-1}\left[\lambda_{n}, \lambda_{n-1}, \ldots, \lambda_{1}\right] \\
f\left[\lambda_{1} t\right] & t f\left[\lambda_{2} t, \lambda_{1} t\right] & \ldots & t^{n-1} f\left[\lambda_{n} t, \lambda_{n-1} t, \ldots, \lambda_{1} t\right] \\
g_{k+1}\left[\lambda_{1}\right] & g_{k+1}\left[\lambda_{2}, \lambda_{1}\right] & \ldots & g_{k+1}\left[\lambda_{n}, \lambda_{n-1}, \ldots, \lambda_{1}\right] \\
\vdots & \vdots & & \vdots \\
g_{n-1}\left[\lambda_{1}\right] & g_{n-1}\left[\lambda_{2}, \lambda_{1}\right] & \ldots & g_{n-1}\left[\lambda_{n}, \lambda_{n-1}, \ldots, \lambda_{1}\right]
\end{array}\right| .
\end{aligned}
$$

The product $\prod_{1 \leq i<j \leq n}\left(\lambda_{j}-\lambda_{i}\right)$ is a Vandermonde's determinant $W$. Notice, that $g_{p}^{(m)}(0)=p$ !, for $m=p$ and zero for others $m$, thus from Property 1 of divided differences we have

$$
g_{p}\left[\lambda_{m}, \ldots, \lambda_{1}\right]= \begin{cases}\sum_{i_{1}+\cdots+i_{m}=p-m+1} \lambda_{m}^{i_{m}} \cdots \lambda_{1}^{i_{1}}, & p>m-1, \\ 1, & p=m-1, \\ 0, & p<m-1\end{cases}
$$

what shows us the expected form of $\frac{W_{k}(t)}{W}$.

\section{References}

1. Arendt, W., Batty, C.J.K.: Tauberian theorems and stability of one parameter semigroups. Trans. Am. Math. Soc. 306, 837-852 (1988)

2. Borichev, A., Tomilov, Y.: Optimal polynomial decay of functions and operator semigroups. Math. Ann. 347(2), 455-478 (2010)

3. Brumley, W.E.: On the asymptotic behaviour of solutions of differential-difference equations of neutral type. J. Differ. Equ. 7, 175-188 (1970)

4. Hale, J.K., Verduyn Lunel, S.M.: Theory of Functional Differential Equations. Springer, New York (1993)

5. Hale, J.K., Verduyn Lunel, S.M.: Strong stabilization of neutral functional differential equations. IMA J. Math. Control Inf. 19(1/2), 5-23 (2002)

6. Haraux, A.: On the fast solution of evolution equations with a rapidly decaying source term. Math. Control Relat. Fields 1(1), 1-20 (2011)

7. Kolmanovskii, V.B., Nosov, V.R.: Stability of Functional Differential Equations. Academic Press, San Diego (1986)

8. Lyubich, Yu.I., Phong, V.Q.: Asymptotic stability of linear differential equation in Banach space. Stud. Math. 88, 37-42 (1988)

9. Medvedev, D.A., Vlasov, V.V.: Functional-differential equations in Sobolev spaces and related problems of spectral theory. J. Math. Sci. 164(5), 659-841 (2010)

10. Miloslavskii, A.I.: Stability of certain classes of evolution equations. Sib. Math. J. 26, 118-132 (1985)

11. O'Connor, D.A., Tarn, T.J.: On stabilization by state feedback for neutral differential equations. IEEE Trans. Autom. Control AC-28(5), 615-618 (1983)

12. Rabah, R., Sklyar, G.M.: Strong stabilizability for a class of linear time delay systems of neutral type. Zh. Mat. Fiz. Anal. Geom. 11(3), 314-330 (2004) 
13. Rabah, R., Sklyar, G.M.: On a class of strongly stabilizable systems of neutral type. Appl. Math. Lett. 18(4), 463-469 (2005)

14. Rabah, R., Sklyar, G.M.: The analysis of exact controllability of neutral-type systems by the moment problem approach. SIAM J. Control Optim. 46(6), 2148-2181 (2007)

15. Rabah, R., Sklyar, G.M., Rezounenko, A.V.: Stability analysis of neutral type systems in Hilbert space. J. Differ. Equ. 214, 391-428 (2005)

16. Rabah, R., Sklyar, G.M., Rezounenko, A.V.: On strong regular stabilizability for linear neutral type systems. J. Differ. Equ. 245, 569-593 (2008)

17. Sklyar, G.M.: On the maximal asymptotics for linear differential equations in Banach spaces. Taiwan. J. Math. 14(6), 2203-2217 (2010)

18. Sklyar, G.M.: Lack of a maximal asymptotics for linear differential equations in Banach spaces. Dokl. Akad. Nauk 431(4), 464-467 (2010); translation in Dokl. Math. 81(2), 265-267 (2010)

19. Sklyar, G.M., Shirman, V.Ya.: On the asymptotic stability of a linear differential equation in a Banach space. Teor. Funkc. Anal. Prilozh. (Kharkov) 37, 127-132 (1982)

20. Sz.-Nagy, B., Foias, C.: Harmonic Analysis of Operators in the Hilbert Space. Mir, Moscow (1970) [Russian translation]

21. van Neerven, J.: The asymptotic behaviour of semigroups of linear operators. In: Operator Theory Advances and Applications, vol. 88. Birkhäuser, Basel (1996)

22. Verduyn Lunel, S.M., Yakubovich, D.V.: A functional model approach to linear neutral functional differential equations. Integral Equ. Oper. Theory 27, 347-378 (1997)

23. Vlasov, V.V., Wu, J.: Sharp estimates of solutions to neutral equations in Sobolev spaces. Funct. Differ. Equ. 12(3-4), 437-461 (2005) 